\title{
Standardization of Drying Techniques to Develop Ready to Cook Banana Inflorescence
}

\author{
Ankan Das* and R.S. Dhua
}

Department of Post Harvest Technology of Horticultural Crops, Faculty of Horticulture, Bidhan Chandra Krishi Viswavidyalaya, Nadia, West Bengal, 741252, India

*Corresponding author

\section{Keywords \\ Banana \\ inflorescence, \\ Pretreatments, \\ Dehydration, \\ Article Info \\ Accepted: \\ 12 January 2019 \\ Available Online: \\ 10 February 2019}

\begin{abstract}
A B S T R A C T
Banana inflorescence is consumed as a vegetable in many Asian countries, is also an excellent source of various minerals such as magnesium, copper and iron. However using it as a vegetable sometimes becomes very demanding as it is very difficult to remove the bracts and extract out the flowers for cooking. So it becomes very important to use some technology to develop ready to cook banana inflorescence which can be preserved for a long period. Dehydration can successfully used to safeguard a commodity as it reduces the bulk volume by lowering the moisture content and also diminishes fungal attack. But in case of dehydration of banana inflorescence the problem of enzymatic browning due to the activity of polyphenol oxidase (PPO) is very pervasive. Therefore the study was aimed to develop suitable dehydration process of banana inflorescence which would yield attractive dehydrated product with long shelf life. Banana inflorescences were subjected to various pretreatments followed by which dehydration was carried at three different temperatures of $50^{\circ} \mathrm{C}, 55^{\circ} \mathrm{C}$ and $60^{\circ} \mathrm{C}$. Thereafter the dehydrated products were packed in LDPE 50 micron pouches and stored in ambient condition. Observation for different physical and biochemical attributes were taken at $0,30,60$ and 90 days of storage. The study revealed that banana inflorescence pretreated with initially dipping at $0.2 \%$ citric acid followed by hot water blanching for 4 minutes and final dipping at $0.1 \%$ sodium metabisulphite with dehydration done at a temperature of $50^{\circ} \mathrm{C}$ was the most promising, maintaining significant observable attributes throughout the study.
\end{abstract}

\section{Introduction}

The flowers of banana also called as the banana inflorescence (Musa sp.) is a pack house of nutrient reserves which makes it an important consumable product for many. In many countries of the Asian subcontinent like India, Malaysia, Philippines, Indonesia and Sri Lanka it is being consumed as a vegetable (Wickramarachchi and Ranamukhaarachchi,
2005). In the state of West Bengal of India this banana inflorescence is very popular, which is commonly called as 'Mocha' in the Bengali language. The banana inflorescence apart from being utilized as a cooking item can also be converted into various other forms like dehydrated products, pickles and canned fruits. For consuming banana inflorescence as a vegetable it sometime becomes very hectic to remove the bracts and extract the flowers. 
So in order to overcome the difficulties of cooking the study was under taken to develop ready to cook dehydrated banana inflorescence.

Drying of food items is one of the very ancient ant common techniques used to increase the shelf life of the produce. The process of dehydration also helps in reducing the bulk volume to a considerable amount which in turn reduces the cost associated with transportation. Drying and dehydration of agricultural products apart from increasing the storage life by bringing down the chances of decay also helps in saving the capital required for transportation and shipping (Dikbasan, 2007). However aside from these merits of dehydration there are also some demerits. For dehydrated products there is always a problem of nutrient loss by leaching and also the chances of microbial contamination though is reduced but still some infestation does takes place during the storage.

Furthermore during dehydration care has to be taken about the dehydration time, as extended period may cause problem. Longer time used for dehydration is unsuitable for the product as it renders the commodity more susceptible towards microbial contaminations (Kostaropoulos and Saravacos, 1995; ElBeltagy et al., 2007; Akbulut and Durmus, 2009). For banana inflorescence there is another situation which comes up. In this case during the process of dehydration the problem of enzymatic browning is very prevalent which takes place due to the activity of polyphenol oxidase (PPO) and substrate concentration. Various processing steps used prior to dehydration like slicing and cutting ads to the enzymatic browning of the inflorescence (Talburt and Smith, 1987; Huxsoll and Bolin, 1989; Wickramarachchi and Ranamukhaarachchi, 2005) which in turn reduces the appearance quality of the final dried product.
Therefore the present study was undertaken with an objective to standardize suitable drying process for banana inflorescence which not only will increase its post harvest longevity but would also yield attractive ready to cook dehydrated product.

\section{Materials and Methods}

The present investigation was carried out in the Department of Post Harvest Technology of Horticultural Crops under the faculty of Horticulture, Bidhan Chandra Krishi Viswavidyalaya, Nadia West Bengal during the year 2015-2016. The crops were collected from farmer's field present in the villages of 'Satyapole' and 'Asudhi' located at Nadia and North 24 Parganas districts of West Bengal respectively. Storage study and the analytical work were conducted in the laboratory of Post Harvest Technology of Horticultural Crops, Bidhan Chandra Krishi Viswavidyalaya, Mohanpur, Nadia, West Bengal.

The banana inflorescence taken for the study was of 'Kanthali' variety. The bracts were carefully removed and the flower buds were separated. The gynaecium part and the scale were discarded from each flower. The banana flowers were subjected to various pretreatments before drying (initial dipping in water containing chemical treatment + blanching in hot water + dipping in cold water containing chemical treatment) under this experiment.

$\mathbf{T}_{\mathbf{1}}-$ Citric acid $0.2 \%+4$ min blanching + potassium metabisulphite $0.1 \%$

$\mathbf{T}_{\mathbf{2}}-$ Citric acid $0.2 \%+4$ min blanching + sodium metabisulphite $0.1 \%$

$\mathbf{T}_{\mathbf{3}}-$ Citric acid $0.2 \%+4 \mathrm{~min}$ blanching + Water

$\mathbf{T}_{\mathbf{4}}-$ Calcium chloride $0.2 \%+4 \mathrm{~min}$ blanching + potassium metabisulphite $0.1 \%$

$\mathbf{T}_{\mathbf{5}}-$ Calcium chloride $0.2 \%+4 \mathrm{~min}$ blanching + sodium metabisulphite $0.1 \%$ 
$\mathbf{T}_{\mathbf{6}}-$ Calcium chloride $0.2 \%+4 \mathrm{~min}$ blanching + Water

$\mathbf{T}_{7}$ - Sodium chloride $0.2 \%+4$ min blanching + potassium metabisulphite $0.1 \%$

$\mathbf{T}_{\mathbf{8}}$ - Sodium chloride $0.2 \%+4$ min blanching + sodium metabisulphite $0.1 \%$

$\mathbf{T}_{\mathbf{9}}$ - Sodium chloride $0.2 \%+4$ min blanching + Water

$\mathbf{T}_{\mathbf{1 0}}-$ Water +4 min blanching + potassium metabisulphite $0.1 \%$

$\mathbf{T}_{\mathbf{1 1}}$ - Water +4 min blanching + sodium metabisulphite $0.1 \%$

$\mathbf{T}_{\mathbf{1 2}}-$ Water + 4 min blanching + Water

Drying was carried out on three different temperatures of $50^{\circ} \mathrm{C}, \quad 55^{\circ} \mathrm{C}$ and $60^{\circ} \mathrm{C}$ followed by packaging in LDPE 50 micron pouches and storage in ambient temperature. Storage studies on different physical and biochemical parameters viz. moisture content, rehydration ratio, total phenols, flavanoids, antioxidant percentage and fungal estimation were carried on $0,30,60$ and 90 days of storage.

\section{Storage conditions- Ambient storage}

Design of experiment: Two Factorial Completely Randomized Design (Sheoran et al., 1998).

\section{Replication- 2}

\section{Moisture content on dry weight basis}

This parameter was calculated according to a formula (Shipley and Vu, 2002).

\section{Moisture content (dehydrated produce)}

The moisture content of dehydrated produce was determined by oven drying method. Dehydrated samples were further dried in a hot air oven at $121{ }^{\circ} \mathrm{C}$ until the weight of the dried sample become stable (A.O.A.C, 2000).

\section{Radical scavenging activity}

The calculation of Radical scavenging activity (RSA) was done by the help of 2, 2-diphenyl1-picrylhydrazyl (DPPH). The variation of the extract sample with respect to the absorbance was measured in a spectrophotometer at 517 $\mathrm{nm}$. The estimation was done by determining the scavenging ability of the antioxidants against the stable DPPH radical (BrandWilliams et al., 1995).

\section{Total phenols}

The estimation of total phenol content present in the sample was done by the help of FolinCiocalteu reagent. The absorbance was calculated spectrophotometrically against a reagent blank at $760 \mathrm{~nm}$ (Singleton et al., 1999).

The final concentration of the total phenol content present in the samples were exhibited as mg gallic acid equivalents (GAE) per gram of fresh weight.

\section{Total flavonoids}

Estimation of the total flavanoid content of the samples was done according to aluminum chloride method (Zhishen et al., 1999) where absorbance was measured in a spectrophotometer at $510 \mathrm{~nm}$ against a prepared reagent blank.

Finally the total flavonoid content was manifested as mg catechin equivalents (CE) per gram of fresh mass.

\section{Microbial load}

Microbial load or the microorganisms present in the samples were calculated by using standard dilution plate count method (Allen, 1953). 


\section{Results and Discussion}

Treatments under all the temperatures viz. $50^{\circ} \mathrm{C} 55^{\circ} \mathrm{C}$ and $60^{\circ} \mathrm{C}$ suffered from periodic loss of moisture content (Fig. 1, 2 and 3) on basis of dry weight, with the gradual passage of dehydration time. At initial phases of dehydration the loss of moisture content (dry wt. basis) from all the treatments under the different temperatures was very expeditious, which gets stabilized (no further decrease in the value) later with further passage of dehydration time.

During the period of storage the moisture content for all the treatments dehydrated at different temperatures viz. $50^{\circ} \mathrm{C} 55^{\circ} \mathrm{C}$ and $60^{\circ} \mathrm{C}$ increased (Table 1). Treatments dehydrated at $50^{\circ} \mathrm{C}$ showed maximum uptake of moisture throughout the period of storage. Treatments dehydrated at $55^{\circ} \mathrm{C}$ showed lesser values, with least moisture gain was recorded for the treatments dehydrated at $60^{\circ} \mathrm{C}$ at the end of the storage. Among the different treatments banana inflorescence which were initially dipped at $0.2 \%$ citric acid followed by hot water blanching for 4 minutes and then dipped in $0.1 \%$ sodium metabisulphite showed the lowest amount of moisture accumulation.'

The values for rehydration ratio decreased during the period of storage (Table 2). After 90 days of storage maximum rehydration ratio were obtained for different treatments dehydrated at $50^{\circ} \mathrm{C}$ followed by treatments dehydrated at $55^{\circ} \mathrm{C}$ and $60^{\circ} \mathrm{C}$ respectively. Banana inflorescence where initial dipping was done at $0.2 \%$ citric acid followed by hot water blanching for 4 minutes and then dipping at $0.1 \%$ sodium metabisulphite was found the best treatment maintaining maximum value of rehydration ratio.The different biochemical parameters viz. total phenols, flavanoids and antioxidant levels (\% inhibition of DPPH) were highest at 0 days of storage and gradually decreased thereafter
(Table 3, 4 and 5). At initial day of storage treatments dehydrated at temperature of $50^{\circ} \mathrm{C}$ showed the highest biochemical values of $31.39 \mathrm{mg} \mathrm{GAE} / \mathrm{g}$ of total phenol, $2.15 \mathrm{mg}$ $\mathrm{CE} / \mathrm{g}$ of total flavanoid, and $59.22 \%$ of antioxidant activity. This was followed by treatments dehydrated at $55^{\circ} \mathrm{C}$ showing 30.66 mg GAE/g of total phenol, $2.04 \mathrm{mg}$ CE/g of total flavanoid and $51.77 \%$ of antioxidant activity. Treatments dehydrated at $60^{\circ} \mathrm{C}$ provided $27.59 \mathrm{mg}$ GAE/g total phenols, 1.86 $\mathrm{mg} \mathrm{CE} / \mathrm{g}$ total flavanoids and $44.32 \%$ antioxidant activity. However later during the period of storage the content of total phenols, flavanoids and antioxidant levels (\% inhibition of DPPH) was reduced for all the treatments dehydrated at temperature of $55^{\circ} \mathrm{C} / \mathrm{B}_{2}$ and $60^{\circ} \mathrm{C} / \mathrm{B}_{3}$. Treatments dehydrated at $50^{\circ} \mathrm{C}$ showed the best reatinment of total phenols, flavanoids and antioxidant levels (\% inhibition of DPPH) throughout the period of storage. At 90 days, dehydration temperature of $50^{\circ} \mathrm{C}$ with banana inflorescence treated with initial dipping of $0.2 \%$ citric acid followed by hot water blanching for 4 minutes and final dipping in $0.1 \%$ sodium metabisulphite showed the maximum values of total phenols, flavanoids and antioxidant levels (\% inhibition of DPPH). Control, dehydrated at $60^{\circ} \mathrm{C}$ recorded the lowest value for all the biochemical parameters.

With the passage of storage time the fungal infestation (unicellular and filamentous type) for different treatments dehydrated at temperatures of $50^{\circ} \mathrm{C}, \quad 55^{\circ} \mathrm{C}$ and $60^{\circ} \mathrm{C}$ increased (Table 6 and 7). Treatments dehydrated at a temperature of $50^{\circ} \mathrm{C}$ were most affected by the fungal attack followed by treatments under $55^{\circ} \mathrm{C}$ and $60^{\circ} \mathrm{C}$. Banana inflorescence were citric acid of $0.2 \%$ was used for initial dipping followed by 4 minutes of hot water blanching and sodium metabisulphite of $0.1 \%$ for final dipping was found the most effective as here the fungal contamination was less. 
Table.1 Moisture content $(\%)$ of dehydrated banana inflorescence subjected to different temperatures at different days in storage

\begin{tabular}{|c|c|c|c|c|c|c|c|c|c|c|c|c|c|c|c|}
\hline O DAS & $\begin{array}{c}\text { 30 } \\
\text { DAS }\end{array}$ & $50^{0} \mathrm{C} / \mathrm{B}_{1}$ & $55^{0} \mathrm{C} / \mathrm{B}_{2}$ & $60^{0} \mathrm{C} / \mathrm{B}_{3}$ & $\begin{array}{c}\text { Mean } \\
\text { A }\end{array}$ & $\begin{array}{c}\text { 60 } \\
\text { DAS }\end{array}$ & $50^{0} \mathrm{C} / \mathrm{B}_{1}$ & $55^{0} \mathrm{C} / \mathrm{B}_{2}$ & $60^{0} \mathrm{C} / \mathrm{B}_{3}$ & $\begin{array}{c}\text { Mean } \\
\text { A }\end{array}$ & 90 DAS & $50^{0} \mathrm{C} / \mathrm{B}_{1}$ & $55^{0} \mathrm{C} / \mathrm{B}_{2}$ & $60^{0} \mathrm{C} / \mathrm{B}_{3}$ & $\begin{array}{c}\text { Mean } \\
\text { A }\end{array}$ \\
\hline \multirow{4}{*}{$\begin{array}{c}A_{(1-12)} \\
B_{1}=5.42\end{array}$} & $\mathrm{~T}_{1} / \mathrm{A}_{1}$ & 5.66 & 4.92 & 3.79 & 4.79 & $\mathbf{T}_{1} / \mathbf{A}_{1}$ & 5.75 & 5.55 & 3.88 & 5.06 & $\mathbf{T}_{1} / \mathbf{A}_{1}$ & 6.79 & 5.58 & 4.08 & 5.48 \\
\hline & $\mathbf{T}_{2} / \mathbf{A}_{2}$ & 5.52 & 4.86 & 3.75 & 4.71 & $\mathbf{T}_{2} / \mathbf{A}_{2}$ & 5.75 & 5.13 & 3.86 & 4.91 & $\mathbf{T}_{2} / \mathbf{A}_{2}$ & 6.50 & 5.12 & 4.02 & 5.21 \\
\hline & $\mathbf{T}_{\mathbf{3}} / \mathbf{A}_{\mathbf{3}}$ & 5.75 & 5.45 & 4.14 & 5.11 & $\mathbf{T}_{\mathbf{3}} / \mathbf{A}_{\mathbf{3}}$ & 7.02 & 6.58 & 4.23 & 5.94 & $\mathbf{T}_{\mathbf{3}} / \mathbf{A}_{\mathbf{3}}$ & 8.46 & 7.55 & 4.25 & 6.75 \\
\hline & $\mathbf{T}_{4} / \mathbf{A}_{4}$ & 5.69 & 4.97 & 3.85 & 4.83 & $\mathbf{T}_{4} / \mathbf{A}_{4}$ & 6.17 & 5.94 & 3.48 & 5.20 & $T_{4} / A_{4}$ & 6.98 & 6.21 & 4.13 & 5.77 \\
\hline \multirow{5}{*}{$\begin{array}{c}A_{(1-12)} \\
B_{2}= \\
4.15\end{array}$} & $\mathbf{T}_{5} / \mathbf{A}_{5}$ & 5.69 & 4.97 & 3.88 & 4.85 & $\mathbf{T}_{5} / \mathbf{A}_{5}$ & 5.79 & 5.55 & 3.92 & 5.08 & $\mathbf{T}_{5} / \mathbf{A}_{5}$ & 6.92 & 5.59 & 4.09 & 5.53 \\
\hline & $T_{6} / A_{6}$ & 5.82 & 5.45 & 4.26 & 5.17 & $T_{6} / A_{6}$ & 7.02 & 6.76 & 5.31 & 6.36 & $T_{6} / A_{6}$ & 8.73 & 7.65 & 4.83 & 7.07 \\
\hline & $\mathbf{T}_{7} / \mathbf{A}_{7}$ & 5.75 & 5.32 & 3.93 & 5.00 & $\mathbf{T}_{7} / \mathbf{A}_{7}$ & 6.44 & 6.25 & 3.98 & 5.55 & $\mathbf{T}_{7} / \mathbf{A}_{7}$ & 7.35 & 6.72 & 4.09 & 6.05 \\
\hline & $\mathbf{T}_{8} / \mathbf{A}_{8}$ & 5.70 & 5.16 & 3.87 & 4.91 & $\mathbf{T}_{8} / \mathbf{A}_{8}$ & 6.42 & 6.17 & 3.97 & 5.52 & $\mathbf{T}_{8} / \mathbf{A}_{8}$ & 7.35 & 6.67 & 4.14 & 6.05 \\
\hline & $\mathbf{T}_{9} / \mathbf{A}_{9}$ & 5.86 & 5.52 & 4.34 & 5.24 & $\mathbf{T}_{9} / \mathbf{A}_{9}$ & 7.17 & 6.77 & 4.45 & 6.13 & $\mathbf{T}_{9} / \mathbf{A}_{9}$ & 9.19 & 7.83 & 5.36 & 7.46 \\
\hline \multirow{8}{*}{$\begin{array}{c}\mathbf{A}_{(1-12)} \\
\mathbf{B}_{3}=3.75\end{array}$} & $\mathbf{T}_{10} / \mathbf{A}_{10}$ & 5.75 & 5.37 & 3.95 & 5.02 & $\mathrm{~T}_{10} / \mathrm{A}_{10}$ & 6.75 & 6.24 & 3.99 & 5.66 & $\mathbf{T}_{10} / \mathbf{A}_{10}$ & 7.75 & 7.25 & 4.26 & 6.42 \\
\hline & $\mathbf{T}_{11} / \mathbf{A}_{11}$ & 5.75 & 5.32 & 3.95 & 5.00 & $\mathbf{T}_{11} / \mathbf{A}_{11}$ & 6.62 & 6.23 & 3.98 & 5.61 & $\mathbf{T}_{11} / \mathbf{A}_{11}$ & 7.35 & 6.83 & 4.09 & 6.09 \\
\hline & $\mathbf{T}_{12} / \mathbf{A}_{12}$ & 5.89 & 5.52 & 4.53 & 5.31 & $\mathbf{T}_{12} / \mathbf{A}_{12}$ & 7.18 & 6.85 & 4.60 & 6.21 & $\mathbf{T}_{12} / \mathbf{A}_{12}$ & 9.60 & 7.93 & 5.55 & 7.69 \\
\hline & $\begin{array}{c}\text { Mean } \\
\text { B }\end{array}$ & 5.73 & 5.23 & 4.02 & & $\begin{array}{c}\text { Mean } \\
\text { B }\end{array}$ & 6.50 & 6.17 & 4.14 & & $\begin{array}{c}\text { Mean } \\
\text { B }\end{array}$ & 7.74 & 6.74 & 4.40 & \\
\hline & & Factors & C.D. & $\operatorname{SE}(d)$ & SE(m) & & Factors & C.D. & $\mathrm{SE}(\mathrm{d})$ & SE(m) & & Factors & C.D. & $\operatorname{SE}(d)$ & $\mathrm{SE}(\mathrm{m})$ \\
\hline & & Factor(A) & 0.020 & 0.010 & 0.007 & & Factor(A) & 0.300 & 0.147 & 0.104 & & Factor(A) & 0.046 & 0.023 & 0.016 \\
\hline & & Factor(B) & 0.010 & 0.005 & 0.003 & & Factor(B) & 0.150 & 0.074 & 0.052 & & Factor(B) & 0.023 & 0.011 & 0.008 \\
\hline & & $\begin{array}{c}\text { Factor(A } \\
\text { X B) }\end{array}$ & 0.034 & 0.017 & 0.012 & & $\begin{array}{c}\text { Factor(A } \\
\text { X B) }\end{array}$ & N/A & 0.255 & 0.180 & & $\begin{array}{c}\text { Factor(A } \\
\text { X B) }\end{array}$ & 0.080 & 0.039 & 0.028 \\
\hline
\end{tabular}

$\mathbf{A}_{(\mathbf{1 - 1 2})}$ : Treatments $\left[\mathbf{A}_{\mathbf{1}}\left(\mathrm{T}_{1}\right)-\right.$ Citric acid $0.2 \%+4$ min blanching $+\mathrm{K}_{2} \mathrm{~S}_{2} \mathrm{O}_{5} 0.1 \%, \mathbf{A}_{2}\left(\mathrm{~T}_{2}\right)-$ Citric acid $0.2 \%+4$ min blanching $+\mathrm{Na}_{2} \mathrm{~S}_{2} \mathrm{O}_{5} 0.1 \%, \mathbf{A}_{\mathbf{3}}\left(\mathrm{T}_{3}\right)-\mathrm{Citric}_{2}$ acid $0.2 \%+4$ min blanching + Water, $\mathbf{A}_{4}\left(\mathrm{~T}_{4}\right)-\mathrm{CaCl}_{2} 0.2 \%+4$ min blanching $+\mathrm{K}_{2} \mathrm{~S}_{2} \mathrm{O}_{5} 0.1 \%, \mathbf{A}_{5}\left(\mathrm{~T}_{5}\right)-\mathrm{CaCl}_{2} 0.2 \%+4$ min blanching $+\mathrm{Na}_{2} \mathrm{~S}_{2} \mathrm{O}_{5} 0.1 \%, \mathbf{A}_{6}\left(\mathrm{~T}_{6}\right)-\mathrm{CaCl}_{2} 0.2 \%+4$ min blanching + Water, $\mathbf{A}_{7}\left(\mathrm{~T}_{7}\right)-\mathrm{NaCl} 0.2 \%+4$ min blanching $+\mathrm{K}_{2} \mathrm{~S}_{2} \mathrm{O}_{5} 0.1 \%, \mathbf{A}_{8}\left(\mathrm{~T}_{8}\right)-\mathrm{NaCl} 0.2 \%+4 \min$ blanching $+\mathrm{Na}_{2} \mathrm{~S}_{2} \mathrm{O}_{5} 0.1 \%, \mathbf{A}_{9}\left(\mathrm{~T}_{9}\right)-\mathrm{NaCl} 0.2 \%+4 \min$ blanching + Water, $\mathbf{A}_{\mathbf{1 0}}\left(\mathrm{T}_{10}\right)-$ Water +4 min blanching $+\mathrm{K}_{2} \mathrm{~S}_{2} \mathrm{O}_{5} 0.1 \%, \mathbf{A}_{\mathbf{1 1}}\left(\mathrm{T}_{11}\right)-$ Water +4 min blanching $+\mathrm{Na}_{2} \mathrm{~S}_{2} \mathrm{O}_{5} 0.1 \%, \mathbf{A}_{\mathbf{1 2}}\left(\mathrm{T}_{12}\right)-$ Water +4 min blanching + Water]: $\mathbf{B}_{(\mathbf{1 - 3})}$ : Temperatures $\left[\mathbf{B}_{1^{-}}-50^{\circ} \mathrm{C}, \mathbf{B}_{2^{-}} 55^{\circ} \mathrm{C}, \mathbf{B}_{3^{-}} 60^{\circ} \mathrm{C}\right], \mathrm{CD}$ at $5 \%$ 
Table.2 Rehydration ratio of dehydrated banana inflorescence subjected to different temperatures at different days in storage

\begin{tabular}{|c|c|c|c|c|c|c|c|c|c|c|c|c|c|c|c|}
\hline 0 das & 30 das & $50^{0} \mathrm{c} / \mathrm{b}_{1}$ & $55^{0} \mathrm{c} / \mathrm{b}_{2}$ & $60^{0} \mathrm{c} / \mathrm{b}_{3}$ & Mean a & 60 das & $50^{0} \mathrm{c} / \mathrm{b}_{1}$ & $55^{0} \mathrm{c} / \mathrm{b}_{2}$ & $60^{0} \mathrm{c} / \mathrm{b}_{3}$ & Mean a & 90 das & $50^{0} \mathrm{c} / \mathrm{b}_{1}$ & $55^{0} \mathrm{c} / \mathrm{b}_{2}$ & $60^{0} \mathrm{c} / \mathrm{b}_{3}$ & Mean a \\
\hline \multirow{4}{*}{$\begin{array}{c}A_{(1-12)} \\
b_{1}=9.12\end{array}$} & $\mathbf{T}_{1} / \mathbf{a}_{1}$ & 8.88 & 7.92 & 6.87 & 7.89 & $\mathbf{T}_{1} / \mathbf{a}_{1}$ & 8.37 & 7.55 & 6.15 & 7.35 & $\mathbf{T}_{1} / \mathbf{a}_{1}$ & 7.75 & 6.83 & 6.06 & 6.88 \\
\hline & $\mathbf{T}_{2} / \mathbf{a}_{2}$ & 8.86 & 7.95 & 6.90 & 7.90 & $\mathbf{T}_{2} / \mathbf{a}_{2}$ & 8.39 & 7.59 & 6.19 & 7.39 & $\mathbf{T}_{2} / \mathbf{a}_{2}$ & 7.76 & 6.95 & 6.25 & 6.98 \\
\hline & $\mathbf{T}_{\mathbf{3}} / \mathbf{a}_{3}$ & 8.84 & 6.94 & 5.72 & 7.16 & $\mathbf{T}_{\mathbf{3}} / \mathbf{a}_{3}$ & 8.19 & 6.53 & 5.47 & 6.73 & $\mathbf{T}_{3} / \mathbf{a}_{3}$ & 7.41 & 6.32 & 5.27 & 6.33 \\
\hline & $\mathbf{T}_{4} / \mathbf{a}_{4}$ & 8.87 & 7.92 & 6.82 & 7.87 & $\mathbf{T}_{4} / \mathbf{a}_{4}$ & 8.27 & 7.22 & 6.12 & 7.20 & $\mathbf{T}_{4} / \mathbf{a}_{4}$ & 7.72 & 6.80 & 6.05 & 6.85 \\
\hline \multirow{5}{*}{$\begin{array}{c}\mathbf{A}_{(1-12)} \\
\mathbf{B}_{2}= \\
8.97\end{array}$} & $\mathbf{T}_{5} / \mathbf{a}_{5}$ & 8.87 & 7.92 & 6.84 & 7.87 & $\mathbf{T}_{5} / \mathbf{a}_{5}$ & 8.27 & 7.54 & 6.12 & 7.31 & $\mathbf{T}_{5} / \mathbf{a}_{5}$ & 7.76 & 6.81 & 6.04 & 6.87 \\
\hline & $T_{6} / \mathbf{a}_{6}$ & 8.83 & 6.94 & 5.71 & 7.16 & $T_{6} / a_{6}$ & 8.20 & 6.27 & 5.92 & 6.80 & $T_{6} / a_{6}$ & 7.41 & 6.13 & 4.24 & 5.93 \\
\hline & $\mathbf{T}_{7} / \mathbf{a}_{7}$ & 8.85 & 7.02 & 6.82 & 7.56 & $\mathbf{T}_{7} / \mathbf{a}_{7}$ & 8.22 & 6.90 & 6.07 & 7.06 & $\mathbf{T}_{7} / \mathbf{a}_{7}$ & 7.55 & 6.80 & 5.75 & 6.70 \\
\hline & $\mathbf{T}_{8} / \mathbf{a}_{8}$ & 8.87 & 7.17 & 6.82 & 7.62 & $\mathbf{T}_{8} / \mathbf{a}_{8}$ & 8.25 & 7.01 & 6.12 & 7.12 & $\mathbf{T}_{8} / \mathbf{a}_{8}$ & 7.58 & 6.80 & 5.93 & 6.77 \\
\hline & $\mathbf{T}_{9} / \mathbf{a}_{9}$ & 8.83 & 6.94 & 5.71 & 7.16 & $\mathbf{T}_{9} / \mathbf{a}_{9}$ & 7.99 & 6.27 & 5.15 & 6.47 & $\mathbf{T}_{9} / \mathbf{a}_{9}$ & 7.33 & 6.12 & 4.56 & 6.00 \\
\hline \multirow{8}{*}{$\begin{array}{c}A_{(1-12)} \\
b_{3}=8.44\end{array}$} & $\mathbf{T}_{10} / \mathbf{a}_{10}$ & 8.84 & 6.96 & 5.72 & 7.17 & $\mathbf{T}_{10} / \mathbf{a}_{10}$ & 8.20 & 6.90 & 6.00 & 7.03 & $\mathbf{T}_{10} / \mathbf{a}_{10}$ & 7.44 & 6.45 & 5.27 & 6.38 \\
\hline & $\mathbf{T}_{11} / \mathbf{a}_{11}$ & 8.84 & 7.02 & 5.75 & 7.20 & $\mathbf{T}_{11} / \mathbf{a}_{11}$ & 8.22 & 6.90 & 6.02 & 7.04 & $\mathbf{T}_{11} / \mathbf{a}_{11}$ & 7.55 & 6.53 & 5.30 & 6.46 \\
\hline & $\mathbf{T}_{12} / \mathbf{a}_{12}$ & 8.81 & 6.94 & 5.56 & 7.10 & $\mathbf{T}_{12} / \mathbf{a}_{12}$ & 7.92 & 6.01 & 5.12 & 6.35 & $\mathbf{T}_{12} / \mathbf{a}_{12}$ & 7.17 & 5.97 & 4.16 & 5.76 \\
\hline & Mean b & 8.85 & 7.30 & 6.27 & & Mean b & 8.20 & 6.89 & 5.87 & & Mean b & 7.53 & 6.54 & 5.40 & \\
\hline & & Factors & C.d. & $\operatorname{Se}(d)$ & $\operatorname{Se}(\mathbf{m})$ & & Factors & C.d. & $\operatorname{Se}(d)$ & $\operatorname{Se}(\mathbf{m})$ & & Factors & C.d. & $\operatorname{Se}(d)$ & $\operatorname{Se}(\mathbf{m})$ \\
\hline & & Factor(a) & 0.016 & 0.008 & 0.006 & & Factor(a) & 0.038 & 0.019 & 0.013 & & Factor(a) & 0.031 & 0.015 & 0.011 \\
\hline & & Factor(b) & 0.008 & 0.004 & 0.003 & & Factor(b) & 0.019 & 0.009 & 0.007 & & Factor(b) & 0.015 & 0.008 & 0.005 \\
\hline & & $\begin{array}{c}\text { Factor(a } \\
\text { x b) }\end{array}$ & 0.029 & 0.014 & 0.010 & & $\begin{array}{c}\text { Factor(a } \\
\text { x b) }\end{array}$ & 0.066 & 0.033 & 0.023 & & $\begin{array}{c}\text { Factor(a } \\
\text { x b) }\end{array}$ & 0.053 & 0.026 & 0.019 \\
\hline
\end{tabular}

$\mathbf{A}_{(\mathbf{1 - 1 2})}$ : Treatments $\left[\mathbf{A}_{\mathbf{1}}\left(\mathrm{T}_{1}\right)-\right.$ Citric acid $0.2 \%+4$ min blanching $+\mathrm{K}_{2} \mathrm{~S}_{2} \mathrm{O}_{5} 0.1 \%, \mathbf{A}_{\mathbf{2}}\left(\mathrm{T}_{2}\right)-$ Citric acid $0.2 \%+4$ min blanching $+\mathrm{Na}_{2} \mathrm{~S}_{2} \mathrm{O}_{5} 0.1 \%, \mathbf{A}_{3}\left(\mathrm{~T}_{3}\right)-\mathrm{Citric}$ acid $0.2 \%+4$ min blanching + Water, $\mathbf{A}_{4}\left(\mathrm{~T}_{4}\right)-\mathrm{CaCl}_{2} 0.2 \%+4$ min blanching $+\mathrm{K}_{2} \mathrm{~S}_{2} \mathrm{O}_{5} 0.1 \%, \mathbf{A}_{5}\left(\mathrm{~T}_{5}\right)-\mathrm{CaCl}_{2} 0.2 \%+4$ min blanching $+\mathrm{Na}_{2} \mathrm{~S}_{2} \mathrm{O}_{5} 0.1 \%, \mathbf{A}_{\mathbf{6}}\left(\mathrm{T}_{6}\right)-\mathrm{CaCl}_{2} 0.2 \%+4 \min$ blanching + Water, $\mathbf{A}_{7}\left(\mathrm{~T}_{7}\right)-\mathrm{NaCl} 0.2 \%+4$ min blanching $+\mathrm{K}_{2} \mathrm{~S}_{2} \mathrm{O}_{5} 0.1 \%, \mathbf{A}_{8}\left(\mathrm{~T}_{8}\right)-\mathrm{NaCl} 0.2 \%+4$ min blanching $+\mathrm{Na}_{2} \mathrm{~S}_{2} \mathrm{O}_{5} 0.1 \%, \mathbf{A}_{9}\left(\mathrm{~T}_{9}\right)-\mathrm{NaCl} 0.2 \%+4$ min blanching + Water, $\mathbf{A}_{\mathbf{1 0}}\left(\mathrm{T}_{10}\right)-$ Water +4 min blanching $+\mathrm{K}_{2} \mathrm{~S}_{2} \mathrm{O}_{5} 0.1 \%, \mathbf{A}_{\mathbf{1 1}}\left(\mathrm{T}_{11}\right)-$ Water +4 min blanching $+\mathrm{Na}_{2} \mathrm{~S}_{2} \mathrm{O}_{5} 0.1 \%, \mathbf{A}_{\mathbf{1 2}}\left(\mathrm{T}_{12}\right)-$ Water +4 min blanching + Water]: $\mathbf{B}_{(\mathbf{1 - 3})}$ : Temperatures $\left[\mathbf{B}_{1^{-}} 50^{\circ} \mathrm{C}, \mathbf{B}_{2^{-}} 55^{0} \mathrm{C}, \mathbf{B}_{3^{-}} 60^{\circ} \mathrm{C}\right], \mathrm{CD}$ at $5 \%$ 
Table.3 Total content of phenols (mg GAE/g) of dehydrated banana inflorescence subjected to different temperatures at different days in storage

\begin{tabular}{|c|c|c|c|c|c|c|c|c|c|c|c|c|c|c|c|}
\hline O DAS & $\begin{array}{c}30 \\
\text { DAS }\end{array}$ & $50^{0} \mathrm{C} / \mathrm{B}_{1}$ & $55^{0} \mathrm{C} / \mathrm{B}_{2}$ & $60^{\circ} \mathrm{C} / \mathrm{B}_{3}$ & $\begin{array}{c}\text { Mean } \\
\text { A }\end{array}$ & $\begin{array}{c}\text { 60 } \\
\text { DAS }\end{array}$ & $50^{0} \mathrm{C} / \mathrm{B}_{1}$ & $55^{0} \mathrm{C} / \mathrm{B}_{2}$ & $60^{\circ} \mathrm{C} / \mathrm{B}_{3}$ & $\begin{array}{c}\text { Mean } \\
\text { A }\end{array}$ & $\begin{array}{c}90 \\
\text { DAS }\end{array}$ & $50^{0} \mathrm{C} / \mathrm{B}_{1}$ & $55^{0} \mathrm{C} / \mathrm{B}_{2}$ & $60^{0} \mathrm{C} / \mathrm{B}_{3}$ & $\begin{array}{c}\text { Mean } \\
\text { A }\end{array}$ \\
\hline \multirow{4}{*}{$\begin{array}{c}\mathbf{A}_{(1-12)} \\
\mathbf{B}_{1}=31.39\end{array}$} & $\mathbf{T}_{1} / \mathbf{A}_{1}$ & 28.14 & 27.09 & 25.12 & 26.78 & $\mathbf{T}_{1} / \mathbf{A}_{1}$ & 25.60 & 23.88 & 22.93 & 24.14 & $\mathbf{T}_{1} / \mathbf{A}_{1}$ & 20.05 & 19.07 & 16.58 & 18.57 \\
\hline & $\mathbf{T}_{2} / \mathbf{A}_{2}$ & 28.39 & 27.14 & 25.54 & 27.02 & $\mathbf{T}_{2} / \mathbf{A}_{2}$ & 26.79 & 25.59 & 23.39 & 25.25 & $\mathbf{T}_{2} / \mathbf{A}_{2}$ & 20.20 & 19.17 & 17.18 & 18.85 \\
\hline & $\mathbf{T}_{\mathbf{3}} / \mathbf{A}_{\mathbf{3}}$ & 25.89 & 22.82 & 19.48 & 22.73 & $\mathbf{T}_{\mathbf{3}} / \mathbf{A}_{\mathbf{3}}$ & 21.14 & 21.77 & 16.68 & 19.86 & $\mathbf{T}_{\mathbf{3}} / \mathbf{A}_{\mathbf{3}}$ & 14.47 & 13.16 & 10.71 & 12.78 \\
\hline & $\mathbf{T}_{4} / \mathbf{A}_{4}$ & 27.93 & 26.91 & 25.02 & 26.62 & $\mathbf{T}_{4} / \mathbf{A}_{4}$ & 25.04 & 23.39 & 22.03 & 23.49 & $\mathbf{T}_{4} / \mathbf{A}_{4}$ & 17.41 & 17.13 & 15.28 & 16.60 \\
\hline \multirow{5}{*}{$\begin{array}{c}\mathbf{A}_{(1-12)} \\
\mathbf{B}_{2}= \\
30.66\end{array}$} & $\mathbf{T}_{5} / \mathbf{A}_{5}$ & 28.05 & 27.79 & 25.09 & 26.97 & $\mathbf{T}_{5} / \mathbf{A}_{5}$ & 25.13 & 25.08 & 22.65 & 24.29 & $\mathbf{T}_{5} / \mathbf{A}_{5}$ & 17.66 & 17.14 & 15.45 & 16.75 \\
\hline & $T_{6} / A_{6}$ & 25.51 & 22.39 & 18.90 & 22.26 & $\mathbf{T}_{6} / \mathbf{A}_{6}$ & 21.94 & 21.03 & 16.18 & 19.72 & $\mathrm{~T}_{6} / \mathrm{A}_{6}$ & 14.38 & 11.88 & 10.49 & 12.25 \\
\hline & $\mathbf{T}_{7} / \mathbf{A}_{7}$ & 26.28 & 26.11 & 21.17 & 24.52 & $\mathbf{T}_{7} / \mathbf{A}_{7}$ & 24.49 & 22.93 & 20.82 & 22.74 & $\mathbf{T}_{7} / \mathbf{A}_{7}$ & 15.17 & 14.23 & 14.34 & 14.58 \\
\hline & $\mathbf{T}_{8} / \mathbf{A}_{8}$ & 26.62 & 26.54 & 22.23 & 25.13 & $\mathbf{T}_{8} / \mathbf{A}_{8}$ & 24.71 & 23.17 & 20.95 & 22.94 & $\mathbf{T}_{8} / \mathbf{A}_{8}$ & 15.37 & 14.53 & 15.10 & 15.00 \\
\hline & $\mathbf{T}_{\mathbf{9}} / \mathbf{A}_{\mathbf{9}}$ & 25.30 & 22.12 & 18.40 & 21.94 & $\mathbf{T}_{\mathbf{9}} / \mathbf{A}_{9}$ & 20.18 & 19.16 & 16.02 & 18.45 & $\mathbf{T}_{9} / \mathbf{A}_{9}$ & 14.20 & 11.13 & 10.04 & 11.79 \\
\hline \multirow{8}{*}{$\begin{array}{c}\mathbf{A}_{(1-12)} \\
B_{3}=27.59\end{array}$} & $\mathbf{T}_{10} / \mathbf{A}_{10}$ & 26.05 & 23.91 & 20.56 & 23.50 & $\mathbf{T}_{10} / \mathbf{A}_{10}$ & 23.50 & 22.19 & 20.07 & 21.92 & $T_{10} / A_{10}$ & 14.55 & 13.65 & 11.59 & 13.26 \\
\hline & $T_{11} / A_{11}$ & 26.16 & 24.64 & 20.95 & 23.91 & $\mathbf{T}_{11} / \mathbf{A}_{11}$ & 23.80 & 22.48 & 20.58 & 22.29 & $\mathbf{T}_{11} / \mathbf{A}_{11}$ & 15.05 & 13.95 & 11.89 & 13.63 \\
\hline & $\mathbf{T}_{12} / \mathbf{A}_{12}$ & 23.36 & 21.83 & 17.60 & 20.93 & $\mathbf{T}_{12} / \mathbf{A}_{12}$ & 19.12 & 18.05 & 15.47 & 17.54 & $\mathbf{T}_{12} / \mathbf{A}_{12}$ & 13.04 & 10.98 & 8.68 & 10.90 \\
\hline & $\begin{array}{c}\text { Mean } \\
\text { B }\end{array}$ & 26.47 & 24.94 & 21.67 & & $\begin{array}{c}\text { Mean } \\
\text { B }\end{array}$ & 23.45 & 22.39 & 19.81 & & $\begin{array}{c}\text { Mean } \\
\text { B }\end{array}$ & 15.96 & 14.67 & 13.11 & \\
\hline & & Factors & C.D. & SE(d) & SE(m) & & Factors & C.D. & $\operatorname{SE}(d)$ & SE(m) & & Factors & C.D. & $\operatorname{SE}(d)$ & $\mathrm{SE}(\mathrm{m})$ \\
\hline & & Factor(A) & 0.097 & 0.048 & 0.034 & & Factor(A) & 0.560 & 0.275 & 0.195 & & Factor(A) & 0.073 & 0.036 & 0.025 \\
\hline & & Factor(B) & 0.049 & 0.024 & 0.017 & & Factor(B) & 0.280 & 0.138 & 0.097 & & Factor(B) & 0.037 & 0.018 & 0.013 \\
\hline & & $\begin{array}{c}\text { Factor(A } \\
\text { X B) }\end{array}$ & 0.168 & 0.083 & 0.058 & & $\begin{array}{c}\text { Factor(A } \\
\text { X B) }\end{array}$ & 0.971 & 0.477 & 0.337 & & $\begin{array}{c}\text { Factor(A } \\
\text { X B ) }\end{array}$ & 0.127 & 0.062 & 0.044 \\
\hline
\end{tabular}

$\mathbf{A}_{(\mathbf{1}-1)}$ : Treatments $\left[\mathbf{A}_{1}\left(\mathrm{~T}_{1}\right)-\right.$ Citric acid $0.2 \%+4$ min blanching $+\mathrm{K}_{2} \mathrm{~S}_{2} \mathrm{O}_{5} 0.1 \%, \mathbf{A}_{\mathbf{2}}\left(\mathrm{T}_{2}\right)-$ Citric acid $0.2 \%+4$ min blanching $+\mathrm{Na}_{2} \mathrm{~S}_{2} \mathrm{O}_{5} 0.1 \%, \mathbf{A}_{\mathbf{3}}\left(\mathrm{T}_{3}\right)-\mathrm{Citric}$ acid $0.2 \%+4$ min blanching + Water, $\mathbf{A}_{\mathbf{4}}\left(\mathrm{T}_{4}\right)-\mathrm{CaCl}_{2} 0.2 \%+4$ min blanching $+\mathrm{K}_{2} \mathrm{~S}_{2} \mathrm{O}_{5} 0.1 \%, \mathbf{A}_{5}\left(\mathrm{~T}_{5}\right)-\mathrm{CaCl}_{2} 0.2 \%+4$ min blanching $+\mathrm{Na}_{2} \mathrm{~S}_{2} \mathrm{O}_{5} 0.1 \%, \mathbf{A}_{\mathbf{6}}\left(\mathrm{T}_{6}\right)-\mathrm{CaCl}_{2} 0.2 \%+4 \min$ blanching + Water, $\mathbf{A}_{7}\left(\mathrm{~T}_{7}\right)-\mathrm{NaCl} 0.2 \%+4$ min blanching $+\mathrm{K}_{2} \mathrm{~S}_{2} \mathrm{O}_{5} 0.1 \%, \mathbf{A}_{\mathbf{8}}\left(\mathrm{T}_{8}\right)-\mathrm{NaCl} 0.2 \%+4$ min blanching $+\mathrm{Na}_{2} \mathrm{~S}_{2} \mathrm{O}_{5} 0.1 \%, \mathbf{A}_{9}\left(\mathrm{~T}_{9}\right)-\mathrm{NaCl} 0.2 \%+4$ min blanching + Water, $\mathbf{A}_{\mathbf{1 0}}\left(\mathrm{T}_{10}\right)-$ Water +4 min blanching $+\mathrm{K}_{2} \mathrm{~S}_{2} \mathrm{O}_{5} 0.1 \%, \mathbf{A}_{\mathbf{1 1}}\left(\mathrm{T}_{11}\right)-$ Water +4 min blanching $+\mathrm{Na}_{2} \mathrm{~S}_{2} \mathrm{O}_{5} 0.1 \%, \mathbf{A}_{12}\left(\mathrm{~T}_{12}\right)-$ Water +4 min blanching + Water]: $\mathbf{B}_{(\mathbf{1}-3)}$ : Temperatures $\left[\mathbf{B}_{1^{-}} 50^{\circ} \mathrm{C}, \mathbf{B}_{2^{-}} 55^{\circ} \mathrm{C}, \mathbf{B}_{3^{-}} 60^{\circ} \mathrm{C}\right], \mathrm{CD}$ at $5 \%$ 
Table.4 Flavanoid content (mg CE/g) of dehydrated banana inflorescence subjected to different temperatures at different days in storage

\begin{tabular}{|c|c|c|c|c|c|c|c|c|c|c|c|c|c|c|c|}
\hline 0 DAS & $\begin{array}{c}\text { 30 } \\
\text { DAS }\end{array}$ & $50^{0} \mathrm{C} / \mathrm{B}_{1}$ & $55^{0} \mathrm{C} / \mathrm{B}_{2}$ & $60^{0} \mathrm{C} / \mathrm{B}_{3}$ & $\begin{array}{c}\text { Mean } \\
\text { A }\end{array}$ & $\begin{array}{c}\text { 60 } \\
\text { DAS }\end{array}$ & $50^{0} \mathrm{C} / \mathrm{B}_{1}$ & $55^{0} \mathrm{C} / \mathrm{B}_{2}$ & $60^{0} \mathrm{C} / \mathrm{B}_{3}$ & $\begin{array}{c}\text { Mean } \\
\text { A }\end{array}$ & $\begin{array}{c}90 \\
\text { DAS }\end{array}$ & $50^{0} \mathrm{C} / \mathrm{B}_{1}$ & $55^{0} \mathrm{C} / \mathrm{B}_{2}$ & $60^{0} \mathrm{C} / \mathrm{B}_{3}$ & $\begin{array}{c}\text { Mean } \\
\text { A }\end{array}$ \\
\hline \multirow{4}{*}{$\begin{array}{c}A_{(1-12)} \\
B_{1}=2.15\end{array}$} & $\mathbf{T}_{1} / \mathbf{A}_{1}$ & 1.85 & 1.71 & 1.48 & 1.68 & $\mathbf{T}_{1} / \mathbf{A}_{1}$ & 1.42 & 1.36 & 0.87 & 1.22 & $T_{1} / A_{1}$ & 1.29 & 1.09 & 0.78 & 1.05 \\
\hline & $\mathbf{T}_{2} / \mathbf{A}_{2}$ & 1.88 & 1.74 & 1.54 & 1.72 & $\mathbf{T}_{2} / \mathbf{A}_{2}$ & 1.45 & 1.42 & 0.97 & 1.28 & $\mathbf{T}_{2} / \mathbf{A}_{2}$ & 1.29 & 1.16 & 0.97 & 1.14 \\
\hline & $\mathbf{T}_{3} / \mathbf{A}_{3}$ & 1.69 & 1.49 & 1.19 & 1.45 & $\mathbf{T}_{\mathbf{3}} / \mathbf{A}_{3}$ & 1.18 & 1.12 & 0.65 & 0.98 & $\mathbf{T}_{\mathbf{3}} / \mathbf{A}_{\mathbf{3}}$ & 1.13 & 0.73 & 0.38 & 0.74 \\
\hline & $\mathbf{T}_{4} / \mathbf{A}_{4}$ & 1.80 & 1.64 & 1.44 & 1.63 & $\mathbf{T}_{4} / \mathbf{A}_{4}$ & 1.40 & 1.30 & 0.83 & 1.18 & $\mathbf{T}_{4} / \mathbf{A}_{4}$ & 1.24 & 0.93 & 0.50 & 0.89 \\
\hline \multirow{5}{*}{$\begin{array}{c}\mathbf{A}_{(1-12)} \\
B_{2}= \\
2.04\end{array}$} & $\mathbf{T}_{5} / \mathbf{A}_{5}$ & 1.82 & 1.69 & 1.48 & 1.66 & $\mathbf{T}_{5} / \mathbf{A}_{5}$ & 1.40 & 1.32 & 0.83 & 1.18 & $\mathbf{T}_{5} / \mathbf{A}_{5}$ & 1.27 & 1.05 & 0.52 & 0.94 \\
\hline & $T_{6} / A_{6}$ & 1.69 & 1.45 & 1.15 & 1.43 & $T_{6} / A_{6}$ & 1.18 & 1.04 & 0.65 & 0.95 & $T_{6} / A_{6}$ & 0.96 & 0.73 & 0.34 & 0.67 \\
\hline & $\mathbf{T}_{7} / \mathbf{A}_{7}$ & 1.74 & 1.55 & 1.33 & 1.54 & $\mathbf{T}_{7} / \mathbf{A}_{7}$ & 1.32 & 1.23 & 0.73 & 1.09 & $\mathbf{T}_{7} / \mathbf{A}_{7}$ & 1.22 & 0.83 & 0.45 & 0.83 \\
\hline & $\mathbf{T}_{8} / \mathbf{A}_{8}$ & 1.76 & 1.61 & 1.39 & 1.59 & $\mathbf{T}_{8} / \mathbf{A}_{8}$ & 1.34 & 1.26 & 0.76 & 1.12 & $\mathbf{T}_{8} / \mathbf{A}_{8}$ & 1.24 & 0.88 & 0.49 & 0.87 \\
\hline & $\mathbf{T}_{9} / \mathbf{A}_{9}$ & 1.64 & 1.34 & 1.07 & 1.35 & $\mathbf{T}_{9} / \mathbf{A}_{9}$ & 1.13 & 0.92 & 0.62 & 0.89 & $\mathrm{~T}_{9} / \mathrm{A}_{9}$ & 0.93 & 0.70 & 0.34 & 0.66 \\
\hline \multirow{8}{*}{$\begin{array}{c}A_{(1-12)} \\
B_{3}=1.86\end{array}$} & $\mathbf{T}_{10} / \mathbf{A}_{10}$ & 1.73 & 1.52 & 1.21 & 1.48 & $\mathrm{~T}_{10} / \mathrm{A}_{10}$ & 1.22 & 1.17 & 0.69 & 1.02 & $\mathrm{~T}_{10} / \mathrm{A}_{10}$ & 1.20 & 0.76 & 0.41 & 0.79 \\
\hline & $\mathbf{T}_{11} / \mathbf{A}_{11}$ & 1.73 & 1.53 & 1.27 & 1.51 & $\mathbf{T}_{11} / \mathbf{A}_{11}$ & 1.27 & 1.23 & 0.71 & 1.07 & $\mathbf{T}_{11} / \mathbf{A}_{11}$ & 1.20 & 0.79 & 0.45 & 0.81 \\
\hline & $\mathbf{T}_{12} / \mathbf{A}_{12}$ & 1.52 & 1.27 & 0.87 & 1.22 & $\mathbf{T}_{12} / \mathbf{A}_{12}$ & 0.96 & 0.87 & 0.62 & 0.82 & $T_{12} / A_{12}$ & 0.88 & 0.69 & 0.28 & 0.61 \\
\hline & $\begin{array}{c}\text { Mean } \\
\text { B }\end{array}$ & 1.73 & 1.54 & 1.28 & & $\begin{array}{c}\text { Mean } \\
\text { B }\end{array}$ & 1.27 & 1.18 & 0.74 & & $\begin{array}{c}\text { Mean } \\
\text { B }\end{array}$ & 1.15 & 0.86 & 0.49 & \\
\hline & & Factors & C.D. & $\operatorname{SE}(d)$ & SE(m) & & Factors & C.D. & $\operatorname{SE}(d)$ & SE(m) & & Factors & C.D. & SE(d) & SE(m) \\
\hline & & Factor(A) & 0.014 & 0.007 & 0.005 & & Factor(A) & 0.013 & 0.007 & 0.005 & & Factor(A) & 0.011 & 0.005 & 0.004 \\
\hline & & Factor(B) & 0.007 & 0.003 & 0.002 & & Factor(B) & 0.007 & 0.003 & 0.002 & & Factor(B) & 0.005 & 0.003 & 0.002 \\
\hline & & $\begin{array}{c}\text { Factor(A } \\
\text { X B) }\end{array}$ & 0.024 & 0.012 & 0.008 & & $\begin{array}{c}\text { Factor(A } \\
\text { X B) }\end{array}$ & 0.023 & 0.011 & 0.008 & & $\begin{array}{c}\text { Factor(A } \\
\text { X B) }\end{array}$ & 0.019 & 0.009 & 0.007 \\
\hline
\end{tabular}

$\mathbf{A}_{(\mathbf{1 - 1 2})}$ : Treatments $\left[\mathbf{A}_{1}\left(\mathrm{~T}_{1}\right)-\right.$ Citric acid $0.2 \%+4$ min blanching $+\mathrm{K}_{2} \mathrm{~S}_{2} \mathrm{O}_{5} 0.1 \%, \mathbf{A}_{2}\left(\mathrm{~T}_{2}\right)-$ Citric acid $0.2 \%+4$ min blanching $+\mathrm{Na}_{2} \mathrm{~S}_{2} \mathrm{O}_{5} 0.1 \%, \mathbf{A}_{3}\left(\mathrm{~T}_{3}\right)-\mathrm{Citric}$ acid $0.2 \%+4$ min blanching + Water, $\mathbf{A}_{4}\left(\mathrm{~T}_{4}\right)-\mathrm{CaCl}_{2} 0.2 \%+4$ min blanching $+\mathrm{K}_{2} \mathrm{~S}_{2} \mathrm{O}_{5} 0.1 \%, \mathbf{A}_{5}\left(\mathrm{~T}_{5}\right)-\mathrm{CaCl}_{2} 0.2 \%+4$ min blanching $+\mathrm{Na}_{2} \mathrm{~S}_{2} \mathrm{O}_{5} 0.1 \%, \mathbf{A}_{\mathbf{6}}\left(\mathrm{T}_{6}\right)-\mathrm{CaCl}_{2} 0.2 \%+4 \min$ blanching + Water, $\mathbf{A}_{7}\left(\mathrm{~T}_{7}\right)-\mathrm{NaCl} 0.2 \%+4$ min blanching $+\mathrm{K}_{2} \mathrm{~S}_{2} \mathrm{O}_{5} 0.1 \%, \quad \mathbf{A}_{8}\left(\mathrm{~T}_{8}\right)-\mathrm{NaCl} 0.2 \%+4$ min blanching $+\mathrm{Na}_{2} \mathrm{~S}_{2} \mathrm{O}_{5} 0.1 \%, \quad \mathbf{A}_{9}\left(\mathrm{~T}_{9}\right)-\mathrm{NaCl} 0.2 \%+4$ min blanching + Water, $\mathbf{A}_{10}\left(\mathrm{~T}_{10}\right)-$ Water +4 min blanching $+\mathrm{K}_{2} \mathrm{~S}_{2} \mathrm{O}_{5} 0.1 \%, \mathbf{A}_{11}\left(\mathrm{~T}_{11}\right)-$ Water +4 min blanching $+\mathrm{Na}_{2} \mathrm{~S}_{2} \mathrm{O}_{5} 0.1 \%, \mathbf{A}_{12}\left(\mathrm{~T}_{12}\right)-$ Water +4 min blanching + Water $]$ : $\mathbf{B}_{(\mathbf{1}-3)}$ : Temperatures $\left[\mathbf{B}_{1^{-}} 50^{\circ} \mathrm{C}, \mathbf{B}_{2^{-}} 55^{0} \mathrm{C}, \mathbf{B}_{3^{-}} 60^{\circ} \mathrm{C}\right], \mathrm{CD}$ at $5 \%$ 
Table.5 Antioxidant activity (percent inhibition of DPPH) of dehydrated banana inflorescence subjected to different temperatures at different days in storage

\begin{tabular}{|c|c|c|c|c|c|c|c|c|c|c|c|c|c|c|c|}
\hline O DAS & $\begin{array}{c}\text { 30 } \\
\text { DAS }\end{array}$ & $50^{0} \mathrm{C} / \mathrm{B}_{1}$ & $55^{0} \mathrm{C} / \mathrm{B}_{2}$ & $60^{0} \mathrm{C} / \mathrm{B}_{3}$ & $\begin{array}{c}\text { Mean } \\
\text { A }\end{array}$ & $\begin{array}{c}\text { 60 } \\
\text { DAS }\end{array}$ & $50^{0} \mathrm{C} / \mathrm{B}_{1}$ & $55^{0} \mathrm{C} / \mathrm{B}_{2}$ & $60^{0} \mathrm{C} / \mathrm{B}_{3}$ & $\begin{array}{c}\text { Mean } \\
\text { A }\end{array}$ & $\begin{array}{c}90 \\
\text { DAS }\end{array}$ & $50^{0} \mathrm{C} / \mathrm{B}_{1}$ & $55^{0} \mathrm{C} / \mathrm{B}_{2}$ & $60^{0} \mathrm{C} / \mathrm{B}_{3}$ & $\begin{array}{c}\text { Mean } \\
\text { A }\end{array}$ \\
\hline \multirow{4}{*}{$\begin{array}{c}\mathbf{A}_{(1-12)} \\
\mathbf{B}_{1}=59.22\end{array}$} & $\mathbf{T}_{1} / \mathbf{A}_{1}$ & 51.18 & 46.92 & 31.90 & 43.33 & $\mathbf{T}_{1} / \mathbf{A}_{1}$ & 34.63 & 33.03 & 26.12 & 31.26 & $\mathbf{T}_{1} / \mathbf{A}_{1}$ & 30.16 & 28.85 & 18.06 & 25.69 \\
\hline & $\mathbf{T}_{2} / \mathbf{A}_{2}$ & 51.18 & 47.78 & 32.71 & 43.89 & $\mathbf{T}_{2} / \mathbf{A}_{2}$ & 37.45 & 33.26 & 26.70 & 32.47 & $\mathbf{T}_{2} / \mathbf{A}_{2}$ & 31.52 & 29.77 & 18.70 & 26.66 \\
\hline & $\mathbf{T}_{3} / \mathbf{A}_{3}$ & 41.90 & 37.47 & 24.16 & 34.51 & $\mathbf{T}_{3} / \mathbf{A}_{3}$ & 27.17 & 22.90 & 15.02 & 21.70 & $\mathbf{T}_{\mathbf{3}} / \mathbf{A}_{3}$ & 21.72 & 16.69 & 9.71 & 16.04 \\
\hline & $\mathbf{T}_{4} / \mathbf{A}_{4}$ & 47.29 & 45.18 & 27.52 & 39.99 & $\mathbf{T}_{4} / \mathbf{A}_{4}$ & 32.84 & 28.81 & 25.31 & 28.98 & $\mathbf{T}_{4} / \mathbf{A}_{4}$ & 26.72 & 24.52 & 13.63 & 21.62 \\
\hline \multirow{5}{*}{$\begin{array}{c}\mathbf{A}_{(1-12)} \\
\mathbf{B}_{2}= \\
51.77\end{array}$} & $T_{5} / A_{5}$ & 50.71 & 45.78 & 27.97 & 41.48 & $\mathrm{~T}_{5} / \mathrm{A}_{5}$ & 33.88 & 31.49 & 19.93 & 28.43 & $T_{5} / A_{5}$ & 30.06 & 28.65 & 13.91 & 24.21 \\
\hline & $T_{6} / A_{6}$ & 41.49 & 35.36 & 23.34 & 33.40 & $T_{6} / A_{6}$ & 26.49 & 22.06 & 14.25 & 20.93 & $\mathbf{T}_{6} / \mathbf{A}_{6}$ & 21.72 & 16.69 & 8.61 & 15.67 \\
\hline & $\mathbf{T}_{7} / \mathbf{A}_{7}$ & 45.07 & 40.92 & 27.13 & 37.70 & $\mathbf{T}_{7} / \mathbf{A}_{7}$ & 29.08 & 27.57 & 17.68 & 24.78 & $\mathbf{T}_{7} / \mathbf{A}_{7}$ & 23.91 & 22.33 & 13.02 & 19.75 \\
\hline & $\mathbf{T}_{8} / \mathbf{A}_{8}$ & 45.21 & 42.61 & 27.13 & 38.31 & $\mathbf{T}_{8} / \mathbf{A}_{8}$ & 29.50 & 28.21 & 19.29 & 25.66 & $\mathbf{T}_{8} / \mathbf{A}_{8}$ & 26.16 & 24.52 & 13.63 & 21.44 \\
\hline & $\mathbf{T}_{9} / \mathbf{A}_{9}$ & 41.49 & 33.27 & 21.82 & 32.19 & $\mathbf{T}_{9} / \mathbf{A}_{9}$ & 26.04 & 19.73 & 14.25 & 20.01 & $\mathbf{T}_{9} / \mathbf{A}_{9}$ & 20.65 & 16.05 & 7.62 & 14.77 \\
\hline \multirow{8}{*}{$\begin{array}{c}A_{(1-12)} \\
B_{3}=44.32\end{array}$} & $\mathbf{T}_{10} / \mathbf{A}_{10}$ & 43.24 & 38.68 & 22.96 & 34.96 & $\mathbf{T}_{10} / \mathbf{A}_{10}$ & 27.81 & 24.11 & 15.02 & 22.31 & $\mathbf{T}_{10} / \mathbf{A}_{10}$ & 23.34 & 17.57 & 9.80 & 16.90 \\
\hline & $\mathbf{T}_{11} / \mathbf{A}_{11}$ & 44.80 & 40.49 & 25.68 & 36.99 & $\mathbf{T}_{11} / \mathbf{A}_{11}$ & 27.92 & 24.64 & 15.53 & 22.69 & $\mathbf{T}_{11} / \mathbf{A}_{11}$ & 23.78 & 18.81 & 12.88 & 18.49 \\
\hline & $\mathbf{T}_{12} / \mathbf{A}_{12}$ & 37.46 & 30.52 & 21.08 & 29.68 & $\mathbf{T}_{12} / \mathbf{A}_{12}$ & 22.51 & 19.15 & 13.08 & 18.25 & $\mathbf{T}_{12} / \mathbf{A}_{12}$ & 18.92 & 15.56 & 7.05 & 13.84 \\
\hline & $\begin{array}{c}\text { Mean } \\
\text { B }\end{array}$ & 45.08 & 40.41 & 26.11 & & $\begin{array}{c}\text { Mean } \\
\text { B }\end{array}$ & 29.61 & 26.25 & 18.51 & & $\begin{array}{c}\text { Mean } \\
\text { B }\end{array}$ & 24.89 & 21.67 & 12.22 & \\
\hline & & Factors & C.D. & $\operatorname{SE}(d)$ & SE(m) & & Factors & C.D. & SE(d) & SE(m) & & Factors & C.D. & $\operatorname{SE}(d)$ & SE(m) \\
\hline & & Factor(A) & 0.467 & 0.230 & 0.162 & & Factor(A) & 0.174 & 0.086 & 0.061 & & Factor(A) & 0.176 & 0.087 & 0.061 \\
\hline & & Factor(B) & 0.234 & 0.115 & 0.081 & & Factor(B) & 0.087 & 0.043 & 0.030 & & Factor(B) & 0.088 & 0.043 & 0.031 \\
\hline & & $\begin{array}{c}\text { Factor(A } \\
\text { X B) }\end{array}$ & 0.810 & 0.398 & 0.281 & & $\begin{array}{c}\text { Factor(A } \\
\text { X B) }\end{array}$ & 0.302 & 0.148 & 0.105 & & $\begin{array}{c}\text { Factor(A } \\
\text { X B) }\end{array}$ & 0.306 & 0.150 & 0.106 \\
\hline
\end{tabular}

$\mathbf{A}_{(\mathbf{1}-12)}$ : Treatments $\left[\mathbf{A}_{1}\left(\mathrm{~T}_{1}\right)-\right.$ Citric acid $0.2 \%+4$ min blanching $+\mathrm{K}_{2} \mathrm{~S}_{2} \mathrm{O}_{5} 0.1 \%, \mathbf{A}_{\mathbf{2}}\left(\mathrm{T}_{2}\right)-$ Citric acid $0.2 \%+4$ min blanching $+\mathrm{Na}_{2} \mathrm{~S}_{2} \mathrm{O}_{5} 0.1 \%, \mathbf{A}_{\mathbf{3}}\left(\mathrm{T}_{3}\right)-\mathrm{Citric}$ acid $0.2 \%+4$ min blanching + Water, $\mathbf{A}_{4}\left(\mathrm{~T}_{4}\right)-\mathrm{CaCl}_{2} 0.2 \%+4$ min blanching $+\mathrm{K}_{2} \mathrm{~S}_{2} \mathrm{O}_{5} 0.1 \%, \mathbf{A}_{5}\left(\mathrm{~T}_{5}\right)-\mathrm{CaCl}_{2} 0.2 \%+4$ min blanching $+\mathrm{Na}_{2} \mathrm{~S}_{2} \mathrm{O}_{5} 0.1 \%, \mathbf{A}_{6}\left(\mathrm{~T}_{6}\right)-\mathrm{CaCl}_{2} 0.2 \%+4 \min$ blanching + Water, $\mathbf{A}_{7}\left(\mathrm{~T}_{7}\right)-\mathrm{NaCl} 0.2 \%+4$ min blanching $+\mathrm{K}_{2} \mathrm{~S}_{2} \mathrm{O}_{5} 0.1 \%, \mathbf{A}_{\mathbf{8}}\left(\mathrm{T}_{8}\right)-\mathrm{NaCl} 0.2 \%+4$ min blanching $+\mathrm{Na}_{2} \mathrm{~S}_{2} \mathrm{O}_{5} 0.1 \%, \mathbf{A}_{\mathbf{9}}\left(\mathrm{T}_{9}\right)-\mathrm{NaCl} 0.2 \%+4 \min$ blanching + Water, $\mathbf{A}_{10}\left(\mathrm{~T}_{10}\right)-$ Water +4 min blanching $+\mathrm{K}_{2} \mathrm{~S}_{2} \mathrm{O}_{5} 0.1 \%, \mathbf{A}_{11}\left(\mathrm{~T}_{11}\right)-$ Water +4 min blanching $+\mathrm{Na}_{2} \mathrm{~S}_{2} \mathrm{O}_{5} 0.1 \%, \mathbf{A}_{12}\left(\mathrm{~T}_{12}\right)-$ Water +4 min blanching + Water]: $\mathbf{B}_{(\mathbf{1 - 3})}$ : Temperatures $\left[\mathbf{B}_{1^{-}}-50^{\circ} \mathrm{C}, \mathbf{B}_{2^{-}} 55^{\circ} \mathrm{C}, \mathbf{B}_{3^{-}}-60^{\circ} \mathrm{C}\right], \mathrm{CD}$ at $5 \%$ 
Table.6 Populations of unicellular fungi $\left(\mathrm{x} 10^{2} \mathrm{cfu} / \mathrm{g}\right)$ on dehydrated banana inflorescence subjected to different temperatures at different days in storage

\begin{tabular}{|c|c|c|c|c|c|c|c|c|c|c|c|c|c|c|c|}
\hline O DAS & $\begin{array}{c}\text { 30 } \\
\text { DAS }\end{array}$ & $50^{0} \mathrm{C} / \mathrm{B}_{1}$ & $55^{0} \mathrm{C} / \mathrm{B}_{2}$ & $60^{0} \mathrm{C} / \mathrm{B}_{3}$ & $\begin{array}{c}\text { Mean } \\
\text { A }\end{array}$ & $\begin{array}{c}\text { 60 } \\
\text { DAS }\end{array}$ & $50^{0} \mathrm{C} / \mathrm{B}_{1}$ & $55^{0} \mathrm{C} / \mathrm{B}_{2}$ & $60^{0} \mathrm{C} / \mathrm{B}_{3}$ & $\begin{array}{c}\text { Mean } \\
\text { A }\end{array}$ & $\begin{array}{c}90 \\
\text { DAS }\end{array}$ & $50^{0} \mathrm{C} / \mathrm{B}_{1}$ & $55^{0} \mathrm{C} / \mathrm{B}_{2}$ & $60^{0} \mathrm{C} / \mathrm{B}_{3}$ & $\begin{array}{c}\text { Mean } \\
\text { A }\end{array}$ \\
\hline \multirow{4}{*}{$\begin{array}{c}\mathbf{A}_{(1-12)} \\
\mathbf{B}_{1}=1.00\end{array}$} & $\mathrm{~T}_{1} / \mathrm{A}_{1}$ & 1.00 & 1.00 & 0.50 & 0.83 & $\mathbf{T}_{1} / \mathbf{A}_{1}$ & 1.50 & 1.50 & 1.00 & 1.33 & $\mathbf{T}_{1} / \mathbf{A}_{1}$ & 2.00 & 2.00 & 2.00 & 2.00 \\
\hline & $\mathbf{T}_{2} / \mathbf{A}_{2}$ & 1.00 & 1.00 & 0.50 & 0.83 & $\mathbf{T}_{2} / \mathbf{A}_{2}$ & 1.50 & 1.50 & 1.00 & 1.33 & $\mathbf{T}_{2} / \mathbf{A}_{2}$ & 2.00 & 2.00 & 1.50 & 1.83 \\
\hline & $\mathbf{T}_{\mathbf{3}} / \mathbf{A}_{\mathbf{3}}$ & 2.00 & 1.50 & 1.50 & 1.67 & $\mathbf{T}_{\mathbf{3}} / \mathbf{A}_{\mathbf{3}}$ & 2.50 & 2.50 & 2.00 & 2.33 & $\mathbf{T}_{\mathbf{3}} / \mathbf{A}_{\mathbf{3}}$ & 4.00 & 3.00 & 2.50 & 3.17 \\
\hline & $\mathbf{T}_{4} / \mathbf{A}_{4}$ & 1.50 & 1.00 & 0.50 & 1.00 & $\mathbf{T}_{4} / \mathbf{A}_{4}$ & 2.00 & 1.50 & 1.50 & 1.67 & $\mathbf{T}_{4} / \mathbf{A}_{4}$ & 2.50 & 2.50 & 2.00 & 2.33 \\
\hline \multirow{5}{*}{$\begin{array}{c}\mathbf{A}_{(1-12)} \\
\mathbf{B}_{2}= \\
\mathbf{1 . 0 0}\end{array}$} & $\mathbf{T}_{5} / \mathbf{A}_{5}$ & 1.50 & 1.00 & 0.50 & 1.00 & $\mathbf{T}_{5} / \mathbf{A}_{5}$ & 1.50 & 1.50 & 1.00 & 1.33 & $\mathbf{T}_{5} / \mathbf{A}_{5}$ & 2.00 & 2.00 & 2.00 & 2.00 \\
\hline & $T_{6} / A_{6}$ & 2.00 & 1.50 & 1.50 & 1.67 & $T_{6} / A_{6}$ & 2.50 & 2.50 & 2.00 & 2.33 & $\mathbf{T}_{6} / \mathbf{A}_{6}$ & 4.50 & 3.50 & 3.00 & 3.67 \\
\hline & $\mathbf{T}_{7} / \mathbf{A}_{7}$ & 1.50 & 1.50 & 1.00 & 1.33 & $\mathbf{T}_{7} / \mathbf{A}_{7}$ & 2.50 & 2.00 & 1.50 & 2.00 & $\mathbf{T}_{7} / \mathbf{A}_{7}$ & 3.00 & 2.50 & 2.50 & 2.67 \\
\hline & $\mathbf{T}_{8} / \mathbf{A}_{8}$ & 1.50 & 1.00 & 1.00 & 1.17 & $\mathbf{T}_{8} / \mathbf{A}_{8}$ & 2.00 & 1.50 & 1.50 & 1.67 & $\mathbf{T}_{8} / \mathbf{A}_{8}$ & 2.50 & 2.50 & 2.50 & 2.50 \\
\hline & $\mathbf{T}_{9} / \mathbf{A}_{9}$ & 2.00 & 1.50 & 1.50 & 1.67 & $\mathbf{T}_{9} / \mathbf{A}_{9}$ & 2.50 & 2.50 & 2.00 & 2.33 & $\mathbf{T}_{9} / \mathbf{A}_{9}$ & 4.00 & 3.50 & 3.00 & 3.50 \\
\hline \multirow{8}{*}{$\begin{array}{c}\mathbf{A}_{(1-12)} \\
\mathbf{B}_{3}=0.5\end{array}$} & $\mathbf{T}_{10} / \mathbf{A}_{10}$ & 1.50 & 1.50 & 1.50 & 1.50 & $\mathrm{~T}_{10} / \mathrm{A}_{10}$ & 2.50 & 2.00 & 2.00 & 2.17 & $\mathbf{T}_{10} / \mathbf{A}_{10}$ & 3.50 & 3.00 & 2.50 & 3.00 \\
\hline & $\mathbf{T}_{11} / \mathbf{A}_{11}$ & 1.50 & 1.50 & 1.00 & 1.33 & $\mathbf{T}_{11} / \mathbf{A}_{11}$ & 2.50 & 2.00 & 1.50 & 2.00 & $\mathbf{T}_{11} / \mathbf{A}_{11}$ & 3.50 & 3.00 & 2.00 & 2.83 \\
\hline & $\mathrm{T}_{12} / \mathrm{A}_{12}$ & 2.00 & 2.00 & 1.50 & 1.83 & $T_{12} / A_{12}$ & 3.00 & 2.50 & 2.50 & 2.67 & $\mathbf{T}_{12} / \mathbf{A}_{12}$ & 5.00 & 4.00 & 3.50 & 4.17 \\
\hline & $\begin{array}{c}\text { Mean } \\
\text { B }\end{array}$ & 1.58 & 1.33 & 1.04 & & $\begin{array}{c}\text { Mean } \\
\text { B }\end{array}$ & 2.21 & 1.96 & 1.63 & & $\begin{array}{c}\text { Mean } \\
\text { B }\end{array}$ & 3.21 & 2.79 & 2.42 & \\
\hline & & Factors & C.D. & $\operatorname{SE}(d)$ & SE(m) & & Factors & C.D. & SE(d) & $\mathbf{S E}(\mathbf{m})$ & & Factors & C.D. & $\mathrm{SE}(\mathrm{d})$ & SE(m) \\
\hline & & Factor(A) & 0.635 & 0.312 & 0.220 & & Factor(A) & 0.664 & 0.326 & 0.231 & & Factor(A) & 0.554 & 0.272 & 0.192 \\
\hline & & Factor(B) & 0.317 & 0.156 & 0.110 & & Factor(B) & 0.332 & 0.163 & 0.115 & & Factor(B) & 0.277 & 0.136 & 0.096 \\
\hline & & $\begin{array}{c}\text { Factor(A } \\
\text { X B) }\end{array}$ & N/A & 0.540 & 0.382 & & $\begin{array}{c}\text { Factor(A } \\
\text { X B) }\end{array}$ & N/A & 0.565 & 0.400 & & $\begin{array}{c}\text { Factor(A } \\
\text { X B) }\end{array}$ & N/A & 0.471 & 0.333 \\
\hline
\end{tabular}

$\mathbf{A}_{(\mathbf{1 - 1 2})}$ : Treatments $\left[\mathbf{A}_{1}\left(\mathrm{~T}_{1}\right)-\right.$ Citric acid $0.2 \%+4$ min blanching $+\mathrm{K}_{2} \mathrm{~S}_{2} \mathrm{O}_{5} 0.1 \%, \mathbf{A}_{2}\left(\mathrm{~T}_{2}\right)-$ Citric acid $0.2 \%+4$ min blanching $+\mathrm{Na}_{2} \mathrm{~S}_{2} \mathrm{O}_{5} 0.1 \%, \mathbf{A}_{\mathbf{3}}\left(\mathrm{T}_{3}\right)-\mathrm{Citric}_{2}$ acid $0.2 \%+4$ min blanching + Water, $\mathbf{A}_{4}\left(\mathrm{~T}_{4}\right)-\mathrm{CaCl}_{2} 0.2 \%+4$ min blanching $+\mathrm{K}_{2} \mathrm{~S}_{2} \mathrm{O}_{5} 0.1 \%, \mathbf{A}_{5}\left(\mathrm{~T}_{5}\right)-\mathrm{CaCl}_{2} 0.2 \%+4$ min blanching $+\mathrm{Na}_{2} \mathrm{~S}_{2} \mathrm{O}_{5} 0.1 \%, \mathbf{A}_{6}\left(\mathrm{~T}_{6}\right)-\mathrm{CaCl}_{2} 0.2 \%+4 \min$ blanching + Water, $\mathbf{A}_{7}\left(\mathrm{~T}_{7}\right)-\mathrm{NaCl} 0.2 \%+4 \min$ blanching $+\mathrm{K}_{2} \mathrm{~S}_{2} \mathrm{O}_{5} 0.1 \%, \mathbf{A}_{\mathbf{8}}\left(\mathrm{T}_{8}\right)-\mathrm{NaCl} 0.2 \%+4$ min blanching $+\mathrm{Na}_{2} \mathrm{~S}_{2} \mathrm{O}_{5} 0.1 \%, \quad \mathbf{A}_{9}\left(\mathrm{~T}_{9}\right)-\mathrm{NaCl} 0.2 \%+4$ min blanching + Water, $\mathbf{A}_{10}\left(\mathrm{~T}_{10}\right)-$ Water +4 min blanching $+\mathrm{K}_{2} \mathrm{~S}_{2} \mathrm{O}_{5} 0.1 \%, \mathbf{A}_{11}\left(\mathrm{~T}_{11}\right)-$ Water +4 min blanching $+\mathrm{Na}_{2} \mathrm{~S}_{2} \mathrm{O}_{5} 0.1 \%, \mathbf{A}_{12}\left(\mathrm{~T}_{12}\right)-$ Water +4 min blanching + Water] $: \mathbf{B}_{(1-3)}$ : Temperatures $\left[\mathbf{B}_{1^{-}}-50^{\circ} \mathrm{C}, \mathbf{B}_{2^{-}} 55^{\circ} \mathrm{C}, \mathbf{B}_{3^{-}} 60^{\circ} \mathrm{C}\right], \mathrm{CD}$ at $5 \%$ 
Table.7 Populations of filamentous fungi $\left(\mathrm{x} 10^{2} \mathrm{cfu} / \mathrm{g}\right)$ on dehydrated banana inflorescence subjected to different temperatures at different days in storage

\begin{tabular}{|c|c|c|c|c|c|c|c|c|c|c|c|c|c|c|c|}
\hline O DAS & $\begin{array}{c}\text { 30 } \\
\text { DAS }\end{array}$ & $50^{0} \mathrm{C} / \mathrm{B}_{1}$ & $55^{0} \mathrm{C} / \mathrm{B}_{2}$ & $60^{0} \mathrm{C} / \mathrm{B}_{3}$ & $\begin{array}{c}\text { Mean } \\
\text { A }\end{array}$ & $\begin{array}{c}\text { 60 } \\
\text { DAS }\end{array}$ & $50^{0} \mathrm{C} / \mathrm{B}_{1}$ & $55^{0} \mathrm{C} / \mathrm{B}_{2}$ & $60^{0} \mathrm{C} / \mathrm{B}_{3}$ & $\begin{array}{c}\text { Mean } \\
\text { A }\end{array}$ & $\begin{array}{c}90 \\
\text { DAS }\end{array}$ & $50^{0} \mathrm{C} / \mathrm{B}_{1}$ & $55^{0} \mathrm{C} / \mathrm{B}_{2}$ & $60^{0} \mathrm{C} / \mathrm{B}_{3}$ & $\begin{array}{c}\text { Mean } \\
\text { A }\end{array}$ \\
\hline \multirow{4}{*}{$\frac{\mathbf{A}_{(1-12)}}{\mathbf{B}_{1}=0.50}$} & $\mathrm{~T}_{1} / \mathrm{A}_{1}$ & 0.50 & 0.50 & 0.50 & 0.50 & $\mathbf{T}_{1} / \mathbf{A}_{1}$ & 1.50 & 1.00 & 1.00 & 1.17 & $\mathbf{T}_{1} / \mathbf{A}_{1}$ & 1.50 & 1.00 & 1.00 & 1.17 \\
\hline & $\mathbf{T}_{2} / \mathbf{A}_{2}$ & 0.50 & 0.50 & 0.50 & 0.50 & $\mathbf{T}_{2} / \mathbf{A}_{2}$ & 1.00 & 0.50 & 1.00 & 0.83 & $\mathbf{T}_{2} / \mathbf{A}_{2}$ & 1.00 & 0.50 & 1.00 & 0.83 \\
\hline & $\mathbf{T}_{3} / \mathbf{A}_{3}$ & 1.00 & 1.00 & 1.00 & 1.00 & $\mathbf{T}_{\mathbf{3}} / \mathbf{A}_{3}$ & 1.50 & 1.50 & 1.00 & 1.33 & $\mathbf{T}_{\mathbf{3}} / \mathbf{A}_{\mathbf{3}}$ & 2.50 & 2.00 & 1.50 & 2.00 \\
\hline & $\mathbf{T}_{4} / \mathbf{A}_{4}$ & 0.50 & 0.50 & 0.50 & 0.50 & $\mathbf{T}_{4} / \mathbf{A}_{4}$ & 1.50 & 1.50 & 1.00 & 1.33 & $\mathbf{T}_{4} / \mathbf{A}_{4}$ & 1.50 & 1.50 & 1.00 & 1.33 \\
\hline \multirow{5}{*}{$\begin{array}{c}\mathbf{A}_{(1-12)} \\
\mathbf{B}_{2}= \\
\mathbf{0 . 5 0}\end{array}$} & $\mathbf{T}_{5} / \mathbf{A}_{5}$ & 0.50 & 0.50 & 0.50 & 0.50 & $\mathbf{T}_{5} / \mathbf{A}_{5}$ & 1.50 & 1.00 & 1.00 & 1.17 & $\mathbf{T}_{5} / \mathbf{A}_{5}$ & 1.50 & 1.00 & 1.00 & 1.17 \\
\hline & $T_{6} / A_{6}$ & 1.00 & 1.00 & 1.00 & 1.00 & $T_{6} / A_{6}$ & 1.50 & 1.50 & 1.00 & 1.33 & $\mathbf{T}_{6} / \mathbf{A}_{6}$ & 2.50 & 2.50 & 1.50 & 2.17 \\
\hline & $\mathbf{T}_{7} / \mathbf{A}_{7}$ & 1.00 & 0.50 & 0.50 & 0.67 & $\mathbf{T}_{7} / \mathbf{A}_{7}$ & 1.50 & 1.50 & 1.00 & 1.33 & $\mathbf{T}_{7} / \mathbf{A}_{7}$ & 1.50 & 1.50 & 1.00 & 1.33 \\
\hline & $\mathbf{T}_{8} / \mathbf{A}_{8}$ & 1.00 & 0.50 & 0.50 & 0.67 & $\mathbf{T}_{8} / \mathbf{A}_{8}$ & 1.50 & 1.50 & 1.00 & 1.33 & $\mathbf{T}_{8} / \mathbf{A}_{8}$ & 1.50 & 1.50 & 1.00 & 1.33 \\
\hline & $\mathbf{T}_{9} / \mathbf{A}_{9}$ & 1.00 & 1.00 & 1.00 & 1.00 & $\mathbf{T}_{9} / \mathbf{A}_{9}$ & 1.50 & 1.50 & 1.50 & 1.50 & $\mathbf{T}_{9} / \mathbf{A}_{9}$ & 2.50 & 2.50 & 1.50 & 2.17 \\
\hline \multirow{8}{*}{$\begin{array}{c}\mathbf{A}_{(1-12)} \\
\mathbf{B}_{3}=0.50\end{array}$} & $\mathbf{T}_{10} / \mathbf{A}_{10}$ & 1.00 & 1.00 & 0.50 & 0.83 & $\mathbf{T}_{10} / \mathbf{A}_{10}$ & 2.00 & 1.50 & 1.00 & 1.50 & $\mathbf{T}_{10} / \mathrm{A}_{10}$ & 2.00 & 1.50 & 1.50 & 1.67 \\
\hline & $\mathbf{T}_{11} / \mathbf{A}_{11}$ & 1.00 & 0.50 & 0.50 & 0.67 & $\mathbf{T}_{11} / \mathbf{A}_{11}$ & 1.50 & 1.50 & 1.00 & 1.33 & $\mathbf{T}_{11} / \mathbf{A}_{11}$ & 2.00 & 1.50 & 1.50 & 1.67 \\
\hline & $\mathrm{T}_{12} / \mathrm{A}_{12}$ & 1.50 & 1.50 & 1.00 & 1.33 & $\mathrm{~T}_{12} / \mathrm{A}_{12}$ & 1.50 & 1.50 & 1.50 & 1.50 & $\mathbf{T}_{12} / \mathbf{A}_{12}$ & 2.50 & 2.50 & 2.00 & 2.33 \\
\hline & $\begin{array}{c}\text { Mean } \\
\text { B }\end{array}$ & 0.88 & 0.75 & 0.67 & & $\begin{array}{c}\text { Mean } \\
\text { B }\end{array}$ & 1.50 & 1.33 & 1.08 & & $\begin{array}{c}\text { Mean } \\
\text { B }\end{array}$ & 1.88 & 1.63 & 1.29 & \\
\hline & & Factors & C.D. & $\operatorname{SE}(d)$ & $\mathbf{S E}(\mathbf{m})$ & & Factors & C.D. & $\mathrm{SE}(\mathrm{d})$ & SE(m) & & Factors & C.D. & $\operatorname{SE}(d)$ & $\mathbf{S E}(\mathbf{m})$ \\
\hline & & Factor(A) & N/A & 0.312 & 0.220 & & Factor(A) & N/A & 0.319 & 0.226 & & Factor(A) & 0.664 & 0.326 & 0.231 \\
\hline & & Factor(B) & N/A & 0.156 & 0.110 & & Factor(B) & 0.325 & 0.160 & 0.113 & & Factor(B) & 0.332 & 0.163 & 0.115 \\
\hline & & $\begin{array}{c}\text { Factor(A } \\
\text { X B) }\end{array}$ & N/A & 0.540 & 0.382 & & $\begin{array}{c}\text { Factor(A } \\
\text { X B) }\end{array}$ & N/A & 0.553 & 0.391 & & $\begin{array}{c}\text { Factor(A } \\
\text { X B) }\end{array}$ & N/A & 0.565 & 0.400 \\
\hline
\end{tabular}

$\mathbf{A}_{(\mathbf{1 - 1 2})}$ : Treatments $\left[\mathbf{A}_{1}\left(\mathrm{~T}_{1}\right)-\right.$ Citric acid $0.2 \%+4$ min blanching $+\mathrm{K}_{2} \mathrm{~S}_{2} \mathrm{O}_{5} 0.1 \%, \mathbf{A}_{\mathbf{2}}\left(\mathrm{T}_{2}\right)-$ Citric acid $0.2 \%+4$ min blanching $+\mathrm{Na}_{2} \mathrm{~S}_{2} \mathrm{O}_{5} 0.1 \%, \mathbf{A}_{\mathbf{3}}\left(\mathrm{T}_{3}\right)-\mathrm{Citric}_{2}$ acid $0.2 \%+4$ min blanching + Water, $\mathbf{A}_{4}\left(\mathrm{~T}_{4}\right)-\mathrm{CaCl}_{2} 0.2 \%+4 \min$ blanching $+\mathrm{K}_{2} \mathrm{~S}_{2} \mathrm{O}_{5} 0.1 \%, \mathbf{A}_{5}\left(\mathrm{~T}_{5}\right)-\mathrm{CaCl}_{2} 0.2 \%+4$ min blanching $+\mathrm{Na}_{2} \mathrm{~S}_{2} \mathrm{O}_{5} 0.1 \%, \mathbf{A}_{6}\left(\mathrm{~T}_{6}\right)-\mathrm{CaCl}_{2} 0.2 \%+4 \min$ blanching + Water, $\mathbf{A}_{7}\left(\mathrm{~T}_{7}\right)-\mathrm{NaCl} 0.2 \%+4$ min blanching $+\mathrm{K}_{2} \mathrm{~S}_{2} \mathrm{O}_{5} 0.1 \%, \mathbf{A}_{\mathbf{8}}\left(\mathrm{T}_{8}\right)-\mathrm{NaCl} 0.2 \%+4$ min blanching $+\mathrm{Na}_{2} \mathrm{~S}_{2} \mathrm{O}_{5} 0.1 \%, \quad \mathbf{A}_{9}\left(\mathrm{~T}_{9}\right)-\mathrm{NaCl} 0.2 \%+4$ min blanching + Water, $\mathbf{A}_{10}\left(\mathrm{~T}_{10}\right)-$ Water +4 min blanching $+\mathrm{K}_{2} \mathrm{~S}_{2} \mathrm{O}_{5} 0.1 \%, \mathbf{A}_{11}\left(\mathrm{~T}_{11}\right)-$ Water +4 min blanching $+\mathrm{Na}_{2} \mathrm{~S}_{2} \mathrm{O}_{5} 0.1 \%, \mathbf{A}_{12}\left(\mathrm{~T}_{12}\right)-$ Water +4 min blanching + Water]: $\mathbf{B}_{(\mathbf{1 - 3})}$ : Temperatures $\left[\mathbf{B}_{1^{-}}-50^{\circ} \mathrm{C}, \mathbf{B}_{2^{-}}-55^{\circ} \mathrm{C}, \mathbf{B}_{3^{-}}-60^{\circ} \mathrm{C}\right], \mathrm{CD}$ at $5 \%$ 
Fig.1,2,3 Moisture content on the basis of dry weight during dehydration of banana inflorescence at different temperatures
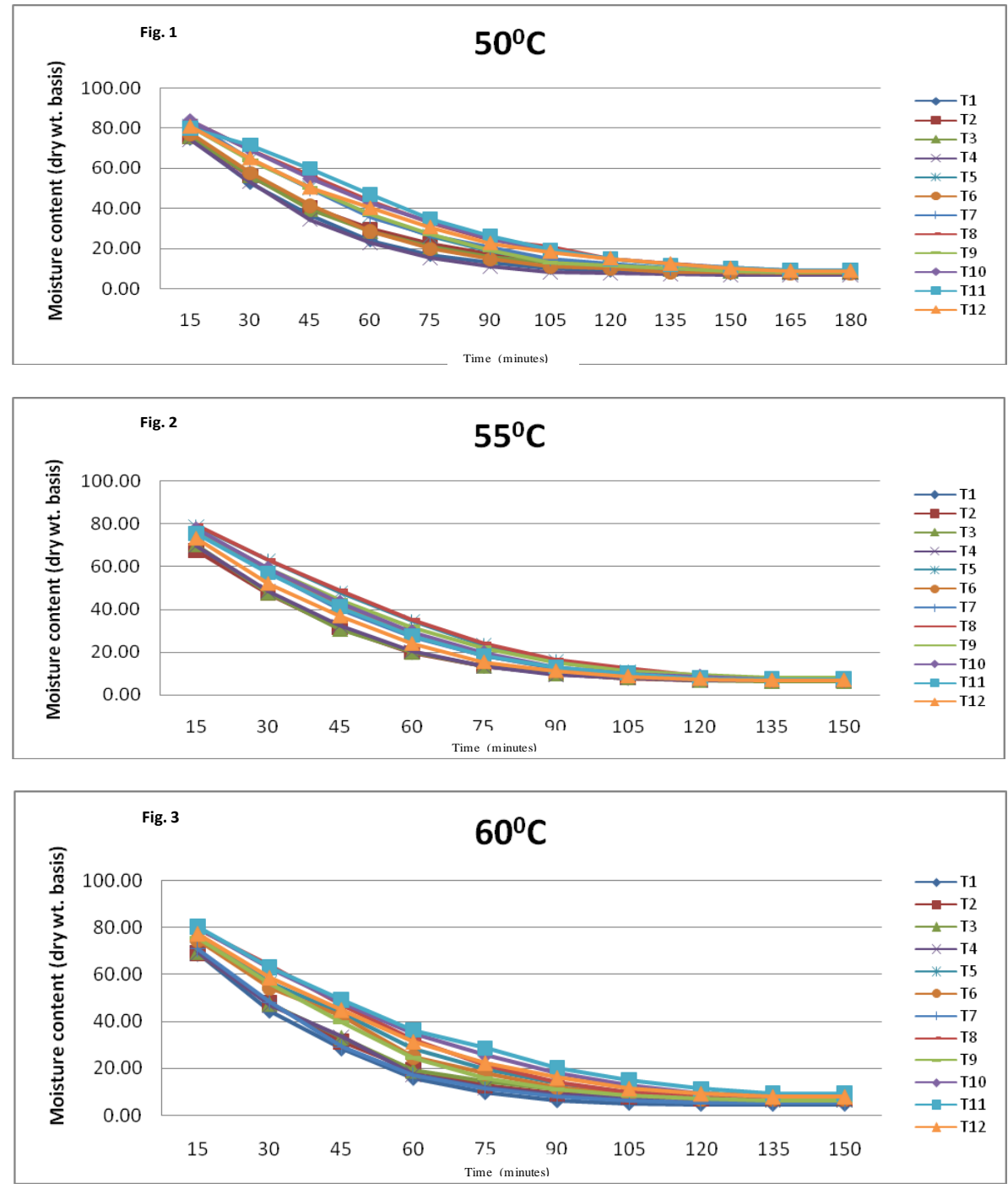

The experiment showed that different physical and biochemical parameters were highest at the initial day of storage for treatments dehydrated at $50^{\circ} \mathrm{C}$ followed by treatments dehydrated at $55^{\circ} \mathrm{C}$ and treatments dehydrated at $60^{\circ} \mathrm{C}$. However later during the period of storage the retainment of different attributes decreased for all the treatments dehydrated at different temperatures.

Prior to dehydration, the banana inflorescence were subjected to various pretreatments in the laboratory conditions. Also the inflorescences of banana were provided with hot water blanching which helped in maintaining the condition of the produce. Blanching was mainly adopted as it helps in loosening and softening of internal tissues which helps in enhancing the rate of drying and facilitates uniform shrinking during dehydration (Kunzek et al., 1999; Munyaka et al., 2010; Waldron et al., 2003).

The chemicals used in the study for treating the banana inflorescence were also found to be very useful. 
Different chemicals which were used for providing pretreatments to banana inflorescence here were citric acid, calcium chloride, sodium chloride, sodium metabisulphite which were also used in the works of Veli et al., 2007; Kostaropoulos and Saravacos, 1995; Kingsly et al., 2007; Doymaz, 2004a,b; El- Beltagy et al., 2007; Pan et al., 2008; Marquez-Rios et al., 2009). These chemicals helped in increasing the post harvest longevity of the dehydrated banana inflorescence. According to Kingsly et al., (2007) pretreatment prior to dehydration helps in inactivation of various enzymes which are responsible for loss of colour they also destress the tissues which minimizes the dehydration time and ultimately provides dehydrated product of superior quality. From the study it was also found that the microbial activity in the post harvest life of the produce got lowered. These pretreatment helped in bringing down the microbial infestations and dehydration furthermore reduces the chances of fungal decay Agbo (2014).

In conclusion, among the three different temperatures used for dehydration, $50^{\circ} \mathrm{C}$ was found best in retaining the physical and biochemical properties of the dehydrated product as compared to the other two temperatures used for dehydration in the study. Though the fungal attack was comparatively little more for treatments dehydrated at $50^{\circ} \mathrm{C}$ then the treatments dehydrated at $55^{\circ} \mathrm{C}$ and $60^{\circ} \mathrm{C}$, but with respect to overall maintenance of physical and biochemical attributes, treatments dehydrated at $50^{\circ} \mathrm{C}$ were found good for storage. Under the dehydration temperature of $50^{\circ} \mathrm{C}$, banana inflorescence initially dipped at $0.2 \%$ citric acid followed by hot water blanching for 4 minutes and then dipped in $0.1 \%$ sodium metabisulphite was most successful in maintaining a significant contents of phenols, flavanoids and antioxidant levels with lesser fungal infestation. Control where banana inflorescence were only dipped in water recorded the lowest value for all the physical and biochemical attributes and showed maximum fungal growth all throughout the storage period.

\section{Acknowledgement}

The first author of the study duly acknowledges the INSPIRE Fellowship Programme under the Department of Science and Technology, Ministry of Science and Technology, New Delhi for continuous financial support during the study.

\section{References}

A.O.A.C. 2000. Official Methods of Analysis. 17th Ed. Association of Official Analytical Chemists, Horwitz, USA.

Agbo, A.E. 2014. Microbiological and nutrional quality of dried okra sold in abidjan markets. Int. J. Sci. Tech. 23 (2): 1585-1600.

Akbulut, A. and Durmus, A. 2009. Thin layer solar drying and mathematical modeling of mulberry. Int. J. Energy Res. 33: 687-695.

Allen, O.N. 1953. Experiments in Soil Bacteriology. Burgess Co., Minneapolis, Minn. pp. 69-70.

Brand-Williams, W., Cuvelier, M.E. and Berset, C. 1995. Use of a free radical method to evaluate antioxidant activity. LWT Food Sci Tech. 28: 25-30.

Dikbasan, T. 2007. Determination of the effective parameters for drying of apples, Master of Science in Energy Engineering (Izmir Institute of Technology, Izmir).

Doymaz, I. 2004a. Effect of pre-treatments using potassium metabisulphide and alkaline ethyl oleate on the drying kinetics of apricots. Biosyst. Eng., 89: 281-287

Doymaz, I. 2004b. Drying kinetics of white mulberry. J. Food Eng. 61: 341-346.

El-Beltagy, A., Gamea, G.R. and Amer Essa, A.H. 2007. Solar drying characteristics of strawberry. J. Food Eng. 78: 456-464.

Huxsoll, C.C. and Bolin, H.R. 1989. Processing 
and distribution alternatives for minimally processed fruits and vegetables. Food Technol. 2: 124-128.

Kingsly, A.R.P., Singh, R., Goyal, R.K. and Singh, D.B. 2007. Thin-layer drying behaviour of organically produced tomato. Am. J. Food Technol. 2: 71-78.

Kostaropoulos, A.E. and Saravacos, G.D. 1995. Microwave pre-treatment for sun-dried raisins. J. Food Sci. 60: 344-347.

Kunzek, H., Kabbert, R. and Gloyna, D. 1999. Aspects of material science in food processing: changes in plant cell walls of fruits and vegetables. Eur. Food Res. Technol. 208(4): 233-250.

Marquez-Rios, E., Ocan ${ }^{\sim}$ o-Higuera, V.M., Maeda-Martinez, A.N., Lugo-Sanchez, M.E., Carvallo-Ruiz, M.G. and PachecoAguilar, R. 2009. Citric acid as pretreatment in drying of Pacific Lion's Paw Scallop (Nodipecten subnodosus) meats. Food Chem. 112: 599-603.

Munyaka, A.W., Oey, I., Van Loey, A. and Hendrickx, M. 2010. Application of thermal inactivation of enzymes during vitamin $\mathrm{C}$ analysis to study the influence of acidification, crushing and blanching on vitamin C stability in Broccoli (Brassica oleracea L var. italica). Food Chem., 120(2): 591-598.

Pan, Z., Shih, C., McHugh, T.H. and Hirschberg, E. 2008. Study of banana dehydration using sequential infrared radiation heating and freeze-drying. Food Sci. Technol. 41: 1944-1951.

Sheoran, O.P., Tonk, D.S., Kaushik, L.S., Hasija, R.C. and Pannu, R.S. (1998).
Statistical Software Package for Agricultural Research Worker. Recent Advances in information theory, Statistics and Computer Applications by D.S. Hooda and R.C. Hasija, Department of Mathematics Statistics, CCS HAU, Hisar (139-143).

Shipley, B. and Vu, T. T. 2002. Dry matter content as a measure of dry matter concentration in plants and their parts. New Phytol. 153, 359-364.

Singleton, V.L., Orthofer, R. and LamuelaRaventos, R.M. 1999. Analysis of total phenols and other oxidation substrates and antioxidants by means of Folin-Ciocalteau reagent. Methods Enzymol. 299: 152-178.

Talburt, W.F and Smith, O. 1987. Potato Processing, 4th ed. Van Nostrand, Reinhold/ AVI, New York.

Veli, D., Bili, M., Tomas, S., Planini, M., Buci'c-Koji, A. and Aladi, K. 2007. Study of the drying kinetics of "Granny Smith" apple in tray drier. Agric. Conspec. Sci. 72: 323-328.

Waldron, K.W., Parker, M.L. and Smith, A.C. 2003. Plant cell wall and food quality: A review. J. Sci. Food Technol. 2:109-10.

Wickramarachchi, K.S., and Ranamukhaarachchi, S.L. (2005). Preservation of Fiber-Rich Banana Blossom as a Dehydrated Vegetable. Sci,Asia. 31: 265-271.

Zhishen, J., Mengcheng, T. and Jianming, W. 1999. The determination of flavonoid contents in mulberry and their scavenging effects on superoxide radicals. Food Chem. 64: 555-59.

\section{How to cite this article:}

Ankan Das and Dhua, R.S. 2019. Standardization of Drying Techniques to Develop Ready to Cook Banana Inflorescence. Int.J.Curr.Microbiol.App.Sci. 8(03): 1523-1536. doi: https://doi.org/10.20546/ijcmas.2019.803.176 